\title{
The Effects of Mixing, Reaction Rates, and Stoichiometry on Yield for Mixing Sensitive Reactions_-Part I: Model Development
}

\author{
Syed Imran A. Shah, ${ }^{1}$ Larry W. Kostiuk, ${ }^{2}$ and Suzanne M. Kresta ${ }^{1}$ \\ ${ }^{1}$ Department of Chemical \& Materials Engineering, University of Alberta, 7th Floor, \\ Electrical \& Computer Engineering Research Facility (ECERF), 9107-116 Street, Edmonton, AB, Canada T6G 2V4 \\ ${ }^{2}$ Department of Mechanical Engineering, University of Alberta, 4-9 Mechanical Engineering Building, \\ Edmonton, $A B$, Canada T6G $2 G 8$ \\ Correspondence should be addressed to Suzanne M. Kresta, suzanne.kresta@ualberta.ca
}

Received 29 February 2012; Accepted 6 April 2012

Academic Editor: Shunsuke Hashimoto

Copyright (๑) 2012 Syed Imran A. Shah et al. This is an open access article distributed under the Creative Commons Attribution License, which permits unrestricted use, distribution, and reproduction in any medium, provided the original work is properly cited.

\begin{abstract}
There are two classes of mixing sensitive reactions: competitive-consecutive and competitive-parallel. The yield of desired product from these coupled reactions depends on how fast the reactants are brought together. Recent experimental results have suggested that the mixing effect may depend strongly on the stoichiometry of the reactions. To investigate this, a $1 \mathrm{D}$, dimensionless, reactiondiffusion model at the micromixing scale was developed. Assuming constant mass concentration and mass diffusivities, systems of PDE's were derived on a mass fraction basis for both types of reactions. Two dimensionless reaction rate ratios and a single general Damköhler number emerged from the analysis. The resulting dimensionless equations were used to investigate the effects of mixing, reaction rate ratio, and reaction stoichiometry. As expected, decreasing either the striation thickness or the dimensionless rate ratio maximizes yield, the reaction stoichiometry has a considerable effect on yield, and all three variables interact strongly.
\end{abstract}

\section{Introduction}

Mixing and apparent reaction rate are intrinsically related: reactions involving multiple reactants cannot occur without the reactants being contacted intimately at a molecular level. In a reactor, reactants are added at the macro- or mesoscale. For the reaction to occur, the pure reactants need to be homogenized at the molecular scale so that molecules can collide. If the mixing is fast enough, the intrinsic chemical kinetics governs the rate of production of new species. This requires a reduction of scale and of differences in concentration, which is the very definition of mixing as it pertains to chemical reactions.

In two known classes of reaction, the progress of the reaction depends heavily on how quickly the reactants are brought together. These reactions consist of two or more competitive reactions either occurring in parallel, where two or more reactions involving the same reactants take place at the same time, or in a consecutive sequence, where the desired product of one of the reactions participates in a second undesired reaction with the original reactants. Both types of reaction schemes can involve considerable production of unwanted by-product despite the desired reaction being as much as a million times faster than the undesired reaction.

Typical representations of these reaction schemes are given in Table 1 . For both cases, $k_{1}^{\prime} \gg k_{2}^{\prime}, P$ is the desired product, and $S$ is the undesired by-product. Therefore, for a perfectly homogeneous mixture of reactants present in a stoichiometric ratio of one $(A: B=1: 1)$, the yield of by-product $S$ should be very small. It is well established (Baldyga and Bourne [1], Patterson et al. [2]) that the yield of byproduct can indeed be quite significant: an effect which is due to imperfect mixing. These classical stoichiometries have been extensively studied (Baldyga and Bourne [1,3], Baldyga et al. [4], Cox et al. [5], Clifford et al. [6], Cox [7], and Patterson et al. [2]). The primary aim of this paper is to study the effect of a different overall reaction stoichiometry on the yield of desired product and the diffusive mass transfer limitations that are associated with this difference. 
TABLE 1: Classic mixing sensitive reaction schemes.

\begin{tabular}{ll}
\hline Competitive-consecutive (C-C) & Competitive-parallel (C-P) \\
\hline$A+B \stackrel{k_{1}^{\prime}}{\longrightarrow} P$ & $A+B \stackrel{k_{1}^{\prime}}{\longrightarrow} P$ \\
$P+B \stackrel{k_{2}^{\prime}}{\longrightarrow} S$ & $C+B \stackrel{k_{2}^{\prime}}{\longrightarrow} S$ \\
\hline
\end{tabular}

The Damköhler number is the dimensionless number used to scale the rate of mixing to the rate of reaction for any given mixing sensitive reaction. There are several forms of the Damköhler number, with the mixing Damköhler number (Da) given by (Patterson et al. [2]):

$$
\mathrm{Da}=\frac{\tau_{M}}{\tau_{R}}=\text { mixing time } \cdot \text { reaction rate }
$$

where $\tau_{M}$ is the characteristic mixing time and $\tau_{R}$ is the characteristic reaction time. The academic and industrial mixing communities have proposed several definitions of $\tau_{M}, \tau_{R}$, and $\mathrm{Da}$ with respect to the reacting flow problem, and some of those efforts are summarized in the following sections.

\section{Literature Review}

The literature review is structured as follows. First, a review of the chemical engineering literature that focuses on competitive-consecutive and competitive-parallel reaction schemes is presented. This is followed by a review of the nonlinear reaction dynamics literature that has focused on the same reaction schemes but from an applied mathematics perspective.

2.1. Mixing Models. Investigations of yield from homogeneous reactions have been investigated by chemical engineers for quite some time starting with Danckwerts $[8,9]$ and Levenspiel [10] who provided analytical solutions for the yield of any reaction provided it is perfectly mixed. Since perfect instantaneous mixing is almost impossible to realise in practice, there have also been a number of investigations into the effect of imperfect mixing on the final yield of desired product (e.g., Patterson et al. [2], Baldyga and Bourne [1, 3], Baldyga et al. [4], and Bhattacharya [11]).

In the fine chemicals industry, reactions are frequently carried out in semi-batch stirred tanks. Mixing and turbulence are very closely related and the rate of mixing is greatly influenced by the turbulence intensity within the tank, which can vary by orders of magnitude in different regions of the tank. The maximum intensity is usually at the impeller, and the minimum is mostly in the bulk of the tank. Therefore, for most mixing sensitive reactions, the reactants are injected at the impeller.

From the more theoretical side, the focus is on a simple geometry with Lagrangian micro-mixing models by Baldyga, Bourne, and others (Baldyga and Pohorecki [12], Baldyga and Bourne [1], and Villermaux and Falk [13]). These are very inexpensive computationally and, in some cases, have analytical solutions. The micro-mixing models have evolved from early alternating striations of reactants to the
Engulfment Model (Baldyga and Bourne [1]) which is widely regarded as the best micro-mixing model currently available. The scales of these models are usually at or below the Kolmogorov scale of turbulent eddies, therefore they are assumed to be independent of the large scale fluid mechanics. The Generalized Mixing Model proposed by Villermaux and Falk [13] is a similar model extended to take into account meso-mixing effects.

There have also been attempts to integrate computational fluid dynamics (CFD) with micro-mixing models. Fox $[14,15]$ combined Villermaux and Falk's [13] Generalized Mixing Model and CFD for turbulent mixing simulations. Muzzio and Liu [16] took a similar approach, integrating a micro-mixing model and CFD for laminar mixing.

The reacting flow problem for multiple competing reactions has also caught the eye of physicists and mathematicians, since it presents interesting non-linear behaviour. A summary of their efforts is given in the next section.

\subsection{Review of the Non-Linear Reaction Dynamics Literature.} The $\mathrm{C}-\mathrm{C}$ reaction provides an interesting non-linear problem that has been extensively investigated by physicists and chaos mathematicians like Cox, Clifford, and others (Clifford [17], Clifford and Cox [18], Clifford et al. [6, 19-21], Cox [7], and Cox et al. [5]). The C-P reaction scheme has been of less interest to the mathematics and physics communities and there is a smaller body of work attached to it (Hecht and Taitelbaum [22], Sinder [23], Sinder et al. [24], and Taitelbaum et al. [25]).

Mixing sensitive reactions exhibit interesting behaviour at reactant interfaces. There are several studies investigating these behaviours for reactions. Cornell and Droz [26] is an example of such a study, where the behaviour of the reaction front for the general single step reaction $m A+n B \rightarrow$ Products was investigated. Cox et al. [5] and later Cox and Finn [27] investigated the reaction interface for the classic C$\mathrm{C}$ reaction extensively. They provided figures and analytical expressions for the profiles of each species at the reaction interface using a model consisting of 1-D alternating reactant striations of varying thickness. The striations had uniform initial concentrations for which they wrote mole balance partial differential equations (PDEs) for each of the species participating in the reaction.

In the long time investigations, Cox et al. [5] first started with stationary and segregated stripes of alternating reactants, that is, "Zebra Stripes," with uniform initial concentrations of reactants across the striations. They performed a mole balance and derived the equations in molar concentrations. Their equations were made dimensionless using the rate constant and concentrations, and the striation thickness was avoided. Their dimensionless equations are:

$$
T=t \cdot\left(k_{1} c_{B_{0}}\right) \quad X=x \cdot\left(\frac{k_{1} c_{B_{0}}}{D}\right)^{1 / 2} \quad I^{\prime}=\frac{c_{I^{\prime}}}{c_{B_{0}}}
$$

where $\left(t, x, c_{I^{\prime}}\right)$ and $\left(T, X, I^{\prime}\right)$ are dimensional and dimensionless time, space, and concentration for species $I^{\prime}$ respectively. $k_{1}$ is the rate constant for the desired reaction, $c_{B_{0}}$ is the initial concentration of the limiting reagent, and $D$ is 
the diffusivity, which was assumed to be equal for all four species $(A, B, R, S)$ involved. Their $R$ is equivalent to $P$ in Table 1 . They used striations of unequal thickness within the same domain so the striation thickness could not be used as a non-dimensionalizing parameter. Since they looked at only one type of stoichiometry, their rate expressions are constant and are the obvious choice for non-dimensionalization.

Initially they looked at a model which had a single striation thickness of initially segregated reactants (Cox et al. [5]). They investigated the effects of initial scale of segregation, that is, striation thicknesses, and the reaction rate ratio for the classic competitive consecutive reaction scheme. They found that decreasing the scale of segregation, that is, striation thickness and the reaction rate ratio $\left(k_{2} / k_{1}\right)$ was favourable. The formulations of the Damköhler number and reaction rate ratio that they found were as follows:

$$
\mathrm{Da}_{\mathrm{II}}=\frac{k_{1} c W^{2}}{D} \quad \varepsilon=\frac{k_{2}}{k_{1}}
$$

where $W$ represents the initial striation thickness of the reactants, $k_{1}$ is the reaction rate of the desired reaction, $c$ is the molar concentration, $D$ is the diffusivity, and $\varepsilon$ is the reaction rate ratio. Using this model, they investigated the yield from zebra stripes of equal thicknesses (Clifford et al. [6]) and confirmed that decreasing the scale of segregation can have a significant favourable effect on the yield of desired product. They also included a parameter to allow for non-stoichiometric initial concentrations of reactants and investigated the effects of having more $A$ than $B$, less $A$ than $B$, and a stoichiometric mixture quite extensively. They also found that if the initial ratio of reactants $(A: B)$ is less than 1 , the yield will go to zero and only if the initial ratio is above 1 can there be a significant yield of desired product.

Clifford [17] and Clifford and Cox [18] took the constant striation thickness model further by assuming a more realistic Gaussian distribution of concentration of reactants within the striations. They compared the full partial differential equations (PDEs) of the Gaussian model with the uniform concentration model and an ordinary differential equation (ODE) model. The uniform concentration model was found to overpredict the yield and the ODE model agreed quite well with the full PDE solution results. This was somewhat of a departure from the rest of the literature on the subject, but provides an interesting perspective on the problem.

The next step Clifford, Cox, and Roberts took was to introduce multiple initial striation thicknesses into the model, the so called "Bar Code" model (Clifford et al. [20, 21]). This gives a closer representation of striation distribution in a chaotic mixing situation. They varied the total number and the arrangement of the striations and found that grouping similar-sized striations together maximized the yield of desired product. Using an average striation thickness for the system over-predicted yield for a small sample, but increasing the number of striations in the model brought the two values closer together.

Clifford et al. [20] investigated the effect of arrangement of striations of alternating reactants $(A$ and $B)$ with varying thicknesses on the yield. The arrangements were chosen such that the widths of the alternating reactants were positively correlated, negatively correlated or placed randomly. Because of the large computational requirements, they applied the Gaussian method developed by Clifford [17]. They found that a positive correlation between the thicknesses of the striations, that is, striations of similar thicknesses grouped together, provided the highest yield of desired product for intermediate times but that there is a crossover at large times where in fact the random arrangement provides the largest final yield of desired product. The negatively correlated case, that is, alternating "thick" and "thin" striations, provided the worst yield. The final step in Clifford, Cox, and Roberts' quest for simulation of reality was to include a stretching parameter to take the reaction-diffusion model to a reaction-diffusionadvection model (Clifford et al. [19]). Cox summarizes these efforts to reduce a chaotic mixing field to a $1 \mathrm{D}$ model, a $2 \mathrm{D}$ model and other reduced models, including various chaotic mixing models, such as the Baker Map model for simulating stretching and folding in [7]. He found that the yield of desired product in a C-C reaction is underestimated by a $1 \mathrm{D}$ lamellar model that ignores the effects of fluid mixing but overestimated by the two other lamellar models (continuous stretching and discrete stretching and folding (Baker Map)) that include the fluid mixing.

All this work was done for the $\mathrm{C}-\mathrm{C}$ reaction scheme and the classic stoichiometry. The C-P reaction scheme has a considerably smaller body of work with most publications concentrating on the reaction front behaviour (Taitelbaum et al. [25], Sinder [23], Sinder et al. [24], and Hecht and Taitelbaum [22]) and the classical stoichiometry.

A Damköhler number for the classic $\mathrm{C}-\mathrm{C}$ reaction was suggested by Cox et al. [5], but there is no such suggestion for a C-C reaction scheme with a general stoichiometry. There is a similar lack of definition for the C-P reaction scheme.

2.3. Mixing and Reaction Rate Ratio. Perfect mixing for reactions is defined as the instant reduction to a homogeneous concentration field. This also corresponds to the perfectly micro-mixed mixing condition (Levenspiel [10]). Good mixing rapidly approaches this perfectly homogeneous condition and hence approaches the maximum yield of desired product.

At the other end of the spectrum is complete segregation of reactants without diffusion and with a minimum surface area for contact. In this case the reaction can occur only at the interface and then comes to a complete halt, so the yield of desired product is minimized. In the absence of diffusion, the only way to increase the yield is to increase the area of contact between reactants, in which case the extent of the reaction is completely dependent on the scale of segregation. The situation is vastly improved with the introduction of diffusion because then reactants away from the interfaces are granted access to one another. Diffusion is the final agent of mixing at the smallest scales of segregation. When the scale of segregation is varied by adjusting the initial striation thickness of the reactants, the limit of perfectly mixed is the case where striation thickness goes to zero, and the limit of perfectly segregated occurs when there is only one interface.

The second aspect of the model is the reaction kinetics. The faster the desired reaction is, compared to the undesired 
reaction, the larger the final yield of desired product will be (Levenspiel [10] and Fogler [28]). Since the objective is to maximize the yield of desired product, a very small $k_{2} / k_{1}$ is favourable and a very large $k_{2} / k_{1}$ is undesirable.

The goal of this paper is to provide some insight to the chemical engineering practitioner who is designing a reactor for a previously uninvestigated mixing sensitive reaction. Prediction of the yield for mixing sensitive reactions has been particularly difficult, as documented in Chapter 13 of the Handbook of Industrial Mixing [2], owing mostly to a lack of information about the reaction schemes, reaction rate ratios, mixing requirements, and so forth. Much of the work on mixing sensitive reactions is for specific known reactions, so the results are not directly transferable to a new reaction, and any general treatment has been restricted to specific stoichiometries, all with coefficients of one. Designs involving more complex reactions often rely on experience and trial and error, or on extensive pilot scale testing.

From the more theoretical point of view, there has been quite a bit of debate on the formulation of the Damköhler number for two stage reactions: does one use the rate of the first reaction, the second reaction, or the reaction for which the information is available? What is the appropriate mixing time? Once a standard Damköhler number can be determined it will be possible to develop a framework around which charts or figures predicting yield for mixing sensitive reactions can be produced, thus making it easier for the practicing chemical engineer to deal with complex reaction systems involving multiple interacting parameters. Even if the model does not serve to predict the yield exactly, it will at least serve to provide a framework for the analysis of new reaction schemes. This paper will improve understanding of the design requirements of reactors for mixing sensitive reactions by clarifying the dominant variables and the interactions between them.

The rest of this paper presents the derivation of a model of the effects of initial mixing condition, reaction rate ratio, and stoichiometry on two types of mixing sensitive reactions: the Competitive-Consecutive $(\mathrm{C}-\mathrm{C})$ reaction and Competitive-Parallel (C-P) reaction.

The key results are:

(1) Development of a model which has a general Damköhler number for any mixing sensitive reaction with a variable stoichiometry.

(2) Tools which allow investigation of the effects of stoichiometry, mixing and relative reaction rates on the final yield of desired product for mixing sensitive reactions of both types: C-C and C-P.

(3) Simulations of the transient behaviour of the reaction interface for short and long times.

\section{Model and Governing Equations}

The model that has been developed is based on an idealized one-dimensional geometry of initially alternating layers of reactants at the micro-mixing scale with a cross section as shown in Figure 1(a). Figure 1(b) depicts an isolated segment of the overall structure in the vicinity of $x=0$, which is placed at the interface between the generic reactant mixtures $Y$ and $Z$, thereby creating a domain of interest bounded by the symmetric zero-flux boundaries at the mid-planes of these layers. In this formulation, the mixtures $Y$ and $Z$ are allowed to take on different species compositions depending on the reaction scheme being considered and the imposed stoichiometry. Figure 1(c) shows the geometry for the specific case of pure striations of $A$ and $B$.

A system of dimensionless reactive-diffusive partial differential equations (PDEs) based on a mass balance was developed for each of the species in the reaction system. It is assumed that the fluid in the system remains homogeneous in phase and is at a constant temperature, as well as being quiescent. Given these assumptions, the general unsteady 1D species mass balance reaction-diffusion equation is given by:

$$
\frac{\partial\left(\rho_{i}\right)}{\partial t}=D_{i} \frac{\partial^{2}\left(\rho_{i}\right)}{\partial x^{2}}+R_{i}
$$

where $\rho_{i}$ is the individual species mass concentration, $D_{i}$ is the individual species diffusivity with respect to the mixture, and $R_{i}$ represents the reaction source/sink terms. $x$ and $t$ are the space and time coordinates, respectively. The model assumes that the initial striation thicknesses of the reactant mixtures are equal, $L_{Z}$, as shown in Figure 1(b) with $L_{Y}=$ $L_{Z}$. One of the objectives of this model is to allow for the investigation of initial mixing conditions by varying the initial striation thicknesses. Space $(x)$ and time $(t)$ are made dimensionless using the initial striation thickness and the molecular diffusivity:

$$
x^{*}=\frac{x}{L_{Z}}=\frac{x}{L_{B}} \quad t^{*}=\frac{t D_{Z}}{L_{Z}^{2}}=\frac{t D_{B}}{L_{B}^{2}}
$$

The choice of $D_{Z}$ and $L_{Z}$ for making the equations dimensionless was made because later on in this paper the composition for the $Z$ layer is to be restricted to a mixture containing only an inert $I$ and/or the limiting reagent $B$, which is always assumed to be the limiting reagent of the reaction regardless of the scheme, effectively making $D_{Z}=$ $D_{B}$ and $L_{Z}=L_{B}$, as shown in (5). The properties of the limiting reagent were used for non-dimensionalization. Species mass concentrations $\left(\rho_{i}\right)$ were converted to mass fractions $\left(w_{i}\right)$ using:

$$
\rho_{T}=\sum \rho_{i} \quad w_{i}=\frac{\rho_{i}}{\rho_{T}}
$$

Using (5)-(6) to modify (4), the dimensionless general species equation for the unsteady 1-D, stationary, reactive-diffusive system is given by:

$$
\frac{\partial\left(w_{i}\right)}{\partial t^{*}}=\frac{D_{i}}{D_{B}} \frac{\partial^{2}\left(w_{i}\right)}{\partial x^{* 2}}+\frac{L_{B}^{2}}{\rho_{T} D_{B}} R_{i}
$$

The assumption of all the species having the same diffusivities was also applied, hence making the coefficient of the elliptical term in (7) unity and giving:

$$
\frac{\partial\left(w_{i}\right)}{\partial t^{*}}=\frac{\partial^{2}\left(w_{i}\right)}{\partial x^{* 2}}+\frac{L_{B}^{2}}{\rho_{T} D_{B}} R_{i}
$$




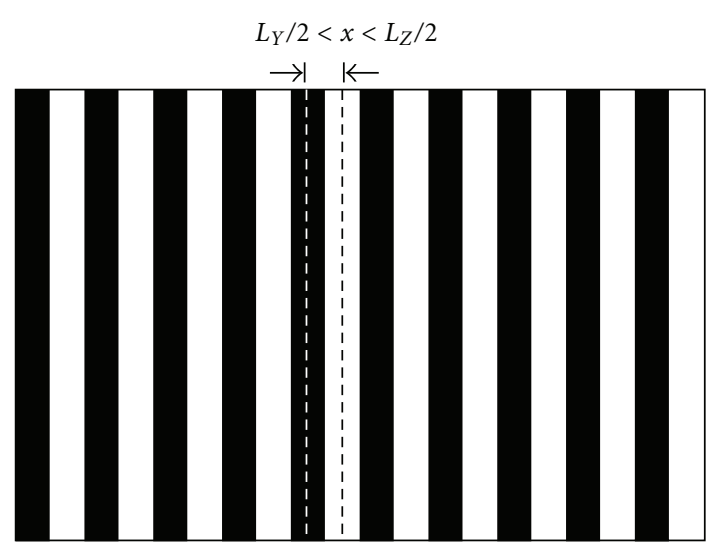

(a)

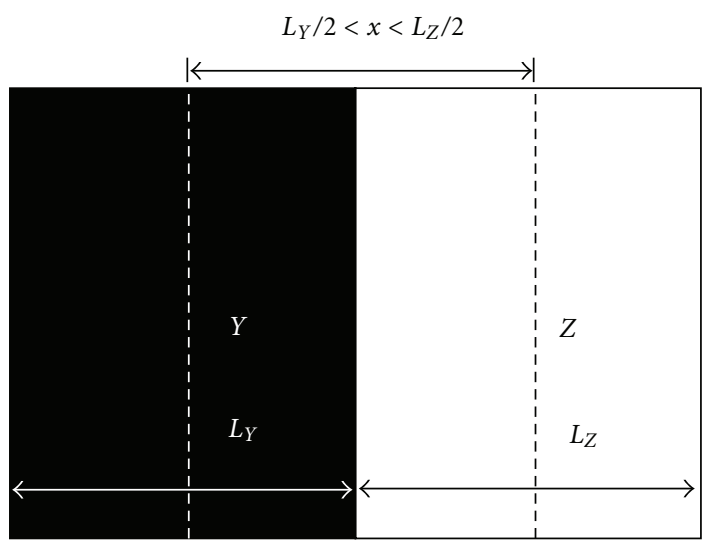

(b)

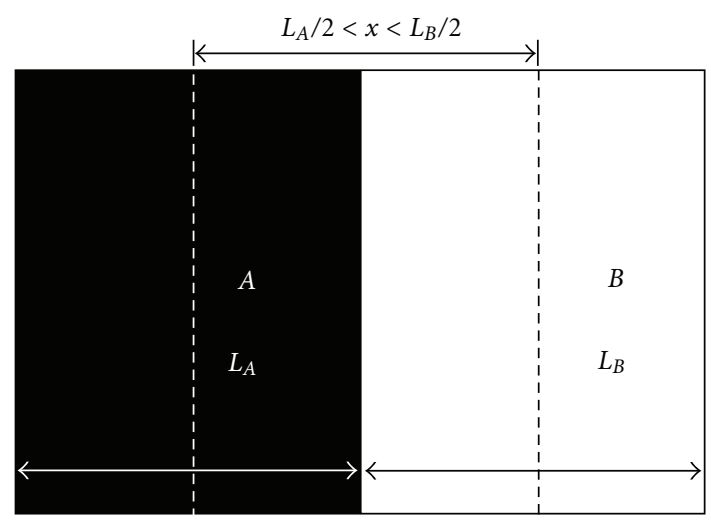

(c)

FIGURE 1: Geometry for proposed mixing model at time $t=0$.

TABLE 2: General mixing sensitive reaction schemes.

\begin{tabular}{ll}
\hline Competitive-consecutive (C-C) & Competitive-parallel (C-P) \\
\hline$A+\epsilon B \stackrel{k_{1}^{\prime}}{\longrightarrow} \alpha P$ & $A+\epsilon B \stackrel{k_{1}^{\prime}}{\longrightarrow} P$ \\
$\beta P+\gamma B \stackrel{k_{2}^{\prime}}{\longrightarrow} S$ & $C+\gamma B \stackrel{k_{2}^{\prime}}{\longrightarrow} S$ \\
\hline
\end{tabular}

Equation (8) represents the reaction-diffusion equation for some arbitrary reaction, represented by the source/sink term $R_{i}$. The particulars of this term define themselves once a reaction scheme is specified. For the purposes of this paper, the reaction scheme will be specified as either a generalized Competitive-Consecutive (C-C) or Competitive-Parallel (C$\mathrm{P})$ reaction between the two layers. For these purposes, layer $Z$ was assumed to be composed of a homogeneous mixture of limiting reagent, $B$, and an inert, $I$, while layer $Y$ was composed of either a single reactant, $A$, or two reactants, $A$ and $C$, again with an inert species mixed into this layer. Table 2 shows the generalized reaction schemes for the two types of mixing sensitive reactions that will be investigated. The effect of species diffusivity was not investigated in this paper.

$A$ and $B$ represent the initial reactants for the $\mathrm{C}-\mathrm{C}$ scheme. $A, B$, and $C$ represent the initial reactants for the C-P scheme. $P$ is the desired product and $S$ the undesired product for both reaction schemes. An inert, $I$, is also present, but it does not participate in the reaction. $k_{1}^{\prime}$ and $k_{2}^{\prime}$ represent the rate constants for the desired and undesired reactions, respectively, and $\alpha, \beta, \gamma, \epsilon$ are the stoichiometric coefficients.

If it is assumed that the reactions are elementary, expressions for $R_{i}$ can be written a priori as molar rate expressions. In order to be used in (8), these molar-based expressions are converted to mass fraction rate expressions using the molecular masses of each species. To keep the focus on the effects of stoichiometry, It was further assumed that the molecular masses of $A, B$, and $C$ were identical $(M)$. As an example, the source term for species $A$ for both $\mathrm{C}-\mathrm{C}$ and $\mathrm{C}-\mathrm{P}$ is given by:

$$
\begin{aligned}
R_{A}\left[\frac{\text { mass }}{m^{3} s}\right] & =-k_{1}^{\prime}[A][B]^{\epsilon} M_{A}=-k_{1}^{\prime} \frac{\rho_{A}}{M_{A}}\left(\frac{\rho_{B}}{M_{B}}\right)^{\epsilon} M_{A} \\
& =-k_{1}^{\prime} \frac{\rho_{T}^{1+\epsilon}}{M_{B}^{\epsilon}} w_{A} w_{B}^{\epsilon}
\end{aligned}
$$

These mass fraction rate expressions are then placed in (8). The molecular masses of $P$ and $S$ depend on the stoichiometry and are derived using the law of mass action. For example, the molecular mass of $P$ for the C-P scheme would be:

$$
M_{P}=M_{A}+\epsilon M_{B}=(1+\epsilon) M
$$


Expressions for the source and sink terms for all participating species can be written as illustrated for species $A$ in (9). It should be noted that the source/sink terms contain all the information for the reaction scheme of interest. The $R_{i}$ terms for the other species are significantly different for the C-C and $\mathrm{C}-\mathrm{P}$ reaction scheme. The systems of partial differential equations (PDE's) are developed separately in the following subsections.

3.1. Competitive-Consecutive (C-C) Reaction Scheme. The $\mathrm{C}-\mathrm{C}$ reaction scheme is the reaction scheme in which the desired product $(P)$, once formed, participates in an undesired reaction with one of the original reactants (in this case, $B)$. The species that participate in the reaction are $A, B$, and $P$. The desired product is $P$ and the undesired by-product is $S$. The general stoichiometry for this type of reaction scheme is given in Table 2. The source and sink term expressions for $A, B, P, S$, and inert $I$ for the C-C reaction scheme are developed by a procedure similar to that shown in (9). Once these expressions are substituted in (8) and simplified, the following system of equations is obtained:

$$
\begin{aligned}
A: \frac{\partial\left(w_{A}\right)}{\partial t^{*}}= & \frac{\partial^{2}\left(w_{A}\right)}{\partial x^{2}}-\left[k_{1}^{\prime}\left(\frac{\rho_{T}}{M}\right)^{\epsilon} \frac{L_{B}^{2}}{D_{B}} w_{A} w_{B}^{\epsilon}\right] \\
B: \frac{\partial\left(w_{B}\right)}{\partial t^{*}}= & \frac{\partial^{2}\left(w_{B}\right)}{\partial x^{2}}-\epsilon\left[k_{1}^{\prime}\left(\frac{\rho_{T}}{M}\right)^{\epsilon} \frac{L_{B}^{2}}{D_{B}} w_{A} w_{B}^{\epsilon}\right] \\
& -\frac{\alpha \gamma}{\beta}\left[\frac{\alpha^{\beta-1} \beta}{(1+\epsilon)^{\beta}} k_{2}^{\prime}\left(\frac{\rho_{T}}{M}\right)^{\beta+\gamma-1} \frac{L_{B}^{2}}{D_{B}} w_{P}^{\beta} w_{B}^{\gamma}\right] \\
P: \frac{\partial\left(w_{P}\right)}{\partial t^{*}}= & \frac{\partial^{2}\left(w_{P}\right)}{\partial x^{* 2}}\left[-\frac{\alpha^{\beta-1} \beta}{(1+\epsilon)^{\beta}} k_{2}^{\prime}\left(\frac{\rho_{T}}{M}\right)^{\beta+\gamma-1} \frac{L_{B}^{2}}{D_{B}} w_{P}^{\beta} w_{B}^{\gamma}\right] \\
& +(1+\epsilon) \\
S: \frac{\partial\left(w_{S}\right)}{\partial t^{*}}= & \frac{\partial^{2}\left(w_{S}\right)}{\partial x^{* 2}+\left(1+\epsilon+\frac{\alpha \gamma}{\beta}\right)} \\
& \times\left[\frac{\alpha^{\beta}-1}{(1+\epsilon)^{\beta}} k_{2}^{\prime}\left(\frac{\rho_{T}}{M}\right)^{\beta+\gamma-1} \frac{L_{B}^{2}}{D_{B}} w_{P}^{\beta} w_{B}^{\gamma}\right] \\
I: \frac{\partial\left(w_{I}\right)}{\partial t^{*}}= & \frac{\partial^{2}\left(w_{I}\right)}{\partial x^{* 2}} w^{\epsilon}
\end{aligned}
$$

In order to compare the effect of the relative rates of the desired and undesired reactions while allowing for different reaction stoichiometries, it is necessary to provide a dimensionless expression for the reaction rate ratio of the desired and undesired reactions. Using the ratio $k_{2}^{\prime} / k_{1}^{\prime}$ is insufficient, since this ratio would have different dimensions for each reaction stoichiometry, making comparison difficult. An ideal dimensionless ratio should give relative rates of the desired reaction to the undesired reaction while retaining a physical meaning that can be intuitively understood. This can be accomplished by comparing the mass conversion rates associated with the first and second reactions. For the $\mathrm{C}-\mathrm{C}$ scheme this was done by comparing the mass rate of consumption of desired product $P$ in the second reaction to the mass rate of production of $P$ in the first reaction:

$$
\frac{k_{2}}{k_{1}}=\frac{\text { mass rate of consumption of } P \text { by undesired reaction }}{\text { mass rate of production of } P \text { by desired reaction }}
$$

The objective is to make this ratio as small as possible to maximize the amount of $P$ produced. By using mass rate expressions to replace the statements in (12) and then simplifying the resulting expression, the dimensionless reaction rate ratio for the general $\mathrm{C}-\mathrm{C}$ reaction scheme becomes:

$$
\frac{k_{2}}{k_{1}}=\left[\frac{\beta}{\alpha}\left(\frac{\alpha}{1+\epsilon}\right)^{\beta}\left(\frac{\rho_{T}}{M}\right)^{\beta+\gamma-\epsilon-1}\right] \frac{k_{2}^{\prime}}{k_{1}^{\prime}}
$$

This physically meaningful $k_{2} / k_{1}$ captures both the effect of stoichiometry and the effect of the reaction rate constants of the two reactions, as well as having the benefit of significantly simplifying (11) to give:

$$
\begin{aligned}
A: \frac{\partial\left(w_{A}\right)}{\partial t^{*}}= & \frac{\partial^{2}\left(w_{A}\right)}{\partial x^{* 2}}-\left[\left(k_{1}^{\prime}\left(\frac{\rho_{T}}{M}\right)^{\epsilon} \frac{L_{B}^{2}}{D_{B}}\right) w_{A} w_{B}^{\epsilon}\right] \\
B: \frac{\partial\left(w_{B}\right)}{\partial t^{*}}= & \frac{\partial^{2}\left(w_{B}\right)}{\partial x^{*}}-\epsilon\left[\left(k_{1}^{\prime}\left(\frac{\rho_{T}}{M}\right)^{\epsilon} \frac{L_{B}^{2}}{D_{B}}\right) w_{A} w_{B}^{\epsilon}\right] \\
& -\frac{\alpha \gamma}{\beta}\left[\left(k_{1}^{\prime}\left(\frac{\rho_{T}}{M}\right)^{\epsilon} \frac{L_{B}^{2}}{D_{B}}\right) \frac{k_{2}}{k_{1}} w_{P}^{\beta} w_{B}^{\gamma}\right] \\
P: \frac{\partial\left(w_{P}\right)}{\partial t^{*}}= & \frac{\partial^{2}\left(w_{P}\right)}{\partial x^{*}} \\
& +(1+\epsilon)\left[\begin{array}{l}
\left(k_{1}^{\prime}\left(\frac{\rho_{T}}{M}\right)^{\epsilon} \frac{L_{B}^{2}}{D_{B}}\right) w_{A} w_{B}^{\epsilon} \\
\left.-\left(k_{1}^{\prime}\left(\frac{\rho_{T}}{M}\right)^{\epsilon} \frac{L_{B}^{2}}{D_{B}}\right) \frac{k_{2}}{k_{1}} w_{P}^{\beta} w_{B}^{\gamma}\right]
\end{array}\right.
\end{aligned}
$$

$$
\begin{aligned}
S: \frac{\partial\left(w_{S}\right)}{\partial t^{*}}= & \frac{\partial^{2}\left(w_{S}\right)}{\partial x^{2}} \\
& +\left(1+\epsilon+\frac{\alpha \gamma}{\beta}\right)\left[\left(k_{1}^{\prime}\left(\frac{\rho_{T}}{M}\right)^{\epsilon} \frac{L_{B}^{2}}{D_{B}}\right) \frac{k_{2}}{k_{1}} w_{P}^{\beta} w_{B}^{\gamma}\right] \\
I: \frac{\partial\left(w_{I}\right)}{\partial t^{*}}= & \frac{\partial^{2}\left(w_{I}\right)}{\partial x^{2}}
\end{aligned}
$$


Examination of (14) shows that there is an expression common to all four of the equations involving reactions, which takes the form of a Damköhler number ( $\mathrm{Da}$ ) given by:

$$
\begin{aligned}
\mathrm{Da} & =k_{1}^{\prime}\left(\frac{\rho_{T}}{M}\right)^{\epsilon} \frac{L_{B}^{2}}{D_{B}}=\frac{k_{1}^{\prime}\left(\rho_{T} / M\right)^{\epsilon}}{\left(D_{B} / L_{B}^{2}\right)} \\
& =\frac{\text { rate of desired fast reaction }}{\text { rate of diffusion }} \\
& =(\text { rate of desired fast reaction }) *(\text { diffusion time })
\end{aligned}
$$

This Da depends on the rate constant of the desired reaction and the initial striation thickness of the reactants. It scales the rate of diffusion at the smallest scale of mixing with the desired reaction rate. The effect of the second reaction rate is included through the rate ratio $k_{2} / k_{1}$. Looking at (15), a small Damköhler number indicates that diffusion in the smallest striation is fast compared to the desired/fast reaction and a large Damköhler number indicates that diffusion is slow compared to the fast reaction. A small $\mathrm{Da}$ is expected to give a high yield.

Cox et al.'s [5] formulations of Damköhler number and dimensionless reaction rate ratio for the classic $\mathrm{C}-\mathrm{C}$ reaction scheme are obtained from our general forms of the Damköhler number (15), and dimensionless reaction rate ratio (13). Setting $\alpha, \beta, \gamma$, and $\epsilon$ equal to 1 gives the classic $\mathrm{C}-\mathrm{C}$ reaction scheme. A factor of 0.5 appears in the $k_{2} / k_{1}$ ratio because we used a mass balance in the derivation of the equations and Cox et al. used a mole balance.

Substituting (15) into (14) gives the final set of equations:

$$
\begin{aligned}
& A: \frac{\partial\left(w_{A}\right)}{\partial t^{*}}=\frac{\partial^{2}\left(w_{A}\right)}{\partial x^{*}}-\left[\mathrm{Da} w_{A} w_{B}^{\epsilon}\right] \\
& B: \frac{\partial\left(w_{B}\right)}{\partial t^{*}}=\frac{\partial^{2}\left(w_{B}\right)}{\partial x^{*}}-\epsilon\left[\mathrm{Da} w_{A} w_{B}^{\epsilon}\right]-\frac{\alpha \gamma}{\beta}\left[\mathrm{Da} \frac{k_{2}}{k_{1}} w_{P}^{\beta} w_{B}^{\gamma}\right] \\
& P: \frac{\partial\left(w_{P}\right)}{\partial t^{*}}=\frac{\partial^{2}\left(w_{P}\right)}{\partial x^{* 2}}+(1+\epsilon)\left[\mathrm{Da} w_{A} w_{B}^{\epsilon}-\mathrm{Da} \frac{k_{2}}{k_{1}} w_{P}^{\beta} w_{B}^{\gamma}\right] \\
& S: \frac{\partial\left(w_{S}\right)}{\partial t^{*}}=\frac{\partial^{2}\left(w_{S}\right)}{\partial x^{2}}+\left(1+\epsilon+\frac{\alpha \gamma}{\beta}\right)\left[\mathrm{Da} \frac{k_{2}}{k_{1}} w_{P}^{\beta} w_{B}^{\gamma}\right] \\
& I: \frac{\partial\left(w_{I}\right)}{\partial t^{*}}=\frac{\partial^{2}\left(w_{I}\right)}{\partial x^{* 2}}
\end{aligned}
$$

Using (16), the effect of reaction rates and striation thickness on $\mathrm{C}-\mathrm{C}$ reactions can be investigated using the dimensionless reaction rate ratio $\left(k_{2} / k_{1}\right)$ and the Damköhler number $(\mathrm{Da})$.

\subsection{Competitive-Parallel (C-P) Reaction Scheme. In the C-P} reaction scheme one of the original reactants (in this case, $B)$ participates in two reactions simultaneously. The general stoichiometry for this type of reaction is shown in Table 2. The source and sink terms for $A, B, C, P, S$, and inert $I$ for the $\mathrm{C}-\mathrm{P}$ reaction scheme were developed following the same procedure as shown for the $\mathrm{C}-\mathrm{C}$ reaction scheme above and then replaced in (8) to get a set of PDE's for the C-P reaction scheme.
As with the $\mathrm{C}-\mathrm{C}$ reaction scheme, variable stoichiometry requires the introduction of a physically meaningful, dimensionless reaction rate ratio for the C-P scheme. Since the C-P reaction scheme has only one reagent $(B)$ which participates in both reactions, the dimensionless reaction rate ratio for the C-P scheme becomes:

$\frac{k_{2}}{k_{1}}=\frac{\text { mass rate of consumption of } B \text { by undesired reaction }}{\text { mass rate of consumption of } B \text { by desired reaction }}$

which, on substitution of rate expressions, can be written as:

$$
\frac{k_{2}}{k_{1}}=\left[\frac{\gamma}{\epsilon}\left(\frac{\rho_{T}}{M}\right)^{\gamma-\epsilon}\right] \frac{k_{2}^{\prime}}{k_{1}^{\prime}}
$$

As with the $k_{2} / k_{1}$ ratio defined for the $\mathrm{C}$ - $\mathrm{C}$ reaction scheme, minimization of this ratio would yield the maximum desirable product $(P)$. This $k_{2} / k_{1}$ also includes the effects of stoichiometry and reaction rate constants for the two reactions. Substitution of (18) into the C-P PDEs and simplifying gives the same Damköhler number that appeared in the $\mathrm{C}-\mathrm{C}$ reaction equations, giving the $\mathrm{C}-\mathrm{P}$ equations for numerical simulations:

$$
\begin{aligned}
& A: \frac{\partial\left(w_{A}\right)}{\partial t^{*}}=\frac{\partial^{2}\left(w_{A}\right)}{\partial x^{2}}-\left[\mathrm{Da} w_{A} w_{B}^{\epsilon}\right] \\
& B: \frac{\partial\left(w_{B}\right)}{\partial t^{*}}=\frac{\partial^{2}\left(w_{B}\right)}{\partial x^{2}}-\epsilon\left[\mathrm{Da} w_{A} w_{B}^{\epsilon}\right]-\epsilon\left[\mathrm{Da} \frac{k_{2}}{k_{1}} w_{c} w_{B}^{\gamma}\right] \\
& C: \frac{\partial\left(w_{C}\right)}{\partial t^{*}}=\frac{\partial^{2}\left(w_{C}\right)}{\partial x^{2}}-\frac{\epsilon}{\gamma}\left[\mathrm{Da} \frac{k_{2}}{k_{1}} w_{c} w_{B}^{\gamma}\right] \\
& P: \frac{\partial\left(w_{P}\right)}{\partial t^{*}}=\frac{\partial^{2}\left(w_{P}\right)}{\partial x^{2}}+(1+\epsilon)\left[\mathrm{Da} w_{A} w_{B}^{\epsilon}\right] \\
& S: \frac{\partial\left(w_{S}\right)}{\partial t^{*}}=\frac{\partial^{2}\left(w_{S}\right)}{\partial x^{2}}+\left(\epsilon+\frac{\epsilon}{\gamma}\right)\left[\mathrm{Da} \frac{k_{2}}{k_{1}} w_{c} w_{B}^{\gamma}\right] \\
& I: \frac{\partial\left(w_{I}\right)}{\partial t^{*}}=\frac{\partial^{2}\left(w_{I}\right)}{\partial x^{2}}
\end{aligned}
$$

This formulation for both C-C and C-P schemes allows the use of one Damköhler number to describe the mixing relative to the desired reaction rate. It also provides a physically meaningful dimensionless reaction rate ratio to describe the relative rates of reaction. While there is no explicit expression for the effect of stoichiometry, both of the dimensionless measures include stoichiometric coefficients, showing that both the mixing and the relative reaction rates are affected by the stoichiometry of the reaction scheme.

\section{Numerical Solution of Equations}

The two systems of equations for the C-C (16) and the C$\mathrm{P}$ (19) reaction schemes were solved using COMSOL Multiphysics 3.4, a commercial finite element PDE solver. The 1$\mathrm{D}$ transient convection and diffusion mass transport model was used with the mass fractions for each species specified as independent variables. Elements were specified as Lagrangequadratic. A 1-D geometry line of unit length equally split 
TABle 3: General initial conditions for C-C and C-P reaction schemes at $t^{*}=0$.

\begin{tabular}{|c|c|c|c|c|c|}
\hline & \multicolumn{2}{|c|}{$\mathrm{C}-\mathrm{C}$} & \multicolumn{3}{|c|}{$\mathrm{C}-\mathrm{P}$} \\
\hline & $-1 / 2 \leq x^{*}<0$ & $0 \leq x^{*} \leq 1 / 2$ & & $-1 / 2 \leq x^{*}<0$ & $0 \leq x^{*} \leq 1 / 2$ \\
\hline$A$ & $w_{A_{0}}$ & 0 & $A$ & $w_{A_{0}}$ & 0 \\
\hline$B$ & 0 & $w_{B_{0}}$ & $B$ & 0 & $w_{B_{0}}$ \\
\hline C & - & - & $C$ & $w_{C_{0}}$ & - \\
\hline$P$ & 0 & 0 & $P$ & 0 & 0 \\
\hline$S$ & 0 & 0 & $S$ & 0 & 0 \\
\hline I & $1-w_{A_{0}}$ & $1-w_{B_{0}}$ & $I$ & $1-w_{A_{0}}-w_{C_{0}}$ & $1-w_{B_{0}}$ \\
\hline
\end{tabular}

TABLE 4: Stoichiometric initial conditions based on $w_{B_{0}}$ for C-C and C-P reaction schemes.

\begin{tabular}{lccccc}
\hline & & C-C & & & C-P \\
& $-1 / 2 \leq x^{*}<0$ & $0 \leq x^{*} \leq 1 / 2$ & & $-1 / 2 \leq x^{*}<0$ & $0 \leq x^{*} \leq 1 / 2$ \\
\hline$A$ & $(1 / \epsilon)\left(w_{B_{0}}\right)$ & 0 & $A$ & $(1 / \epsilon)\left(w_{B_{0}}\right)$ & 0 \\
$B$ & 0 & $w_{B_{0}}$ & $B$ & 0 & $w_{B_{0}}$ \\
$C$ & - & - & $C$ & $(1 / \gamma)\left(w_{B_{0}}\right)$ & 0 \\
$S$ & 0 & 0 & $S$ & 0 & 0 \\
$I$ & 0 & 0 & $I$ & $1-(1 / \epsilon+$ & $1-w_{B_{0}}$ \\
\hline
\end{tabular}

into two domains $\left(-1 / 2 \leq x^{*}<0\right.$ and $\left.0 \leq x^{*} \leq 1 / 2\right)$, and a mesh of 2048 equally spaced elements was generated. Boundary conditions (BCs) for all cases were specified as

$$
\begin{gathered}
\frac{\partial\left(w_{i}\right)}{\partial x^{*}}=0 \quad \text { at } x^{*}=-\frac{1}{2}, x^{*}=\frac{1}{2} \\
\forall t^{*}, i=A, B, C, P, S, I
\end{gathered}
$$

The general initial conditions for the two reaction schemes are shown in Table 3. The initial conditions were chosen to replicate the segregated striation condition of the model.

A final constraint imposed on the simulations is that the reactants need to be present in stoichiometric quantities. Using this constraint, it is possible to express the initial mass fractions as a function of the initial mass fraction of the limiting reagent $B\left(w_{B_{0}}\right)$, as shown in Table 4 . For the C-C case, the only reactants present initially are $A$ and $B$. Therefore, the alternating striations would have mass fractions of unity for $A$ and unity for $B$. For the C-P cases, however, there are three initial reactants present. In this model, it is assumed that reactants $A$ and $C$ are well mixed and present in the $Y$ striation and that the limiting reagent $B$ is in the $Z$ striation. The inert species $I$ was allowed to be present in both $Y$ and $Z$ striations as required to maintain constant mass concentrations in the striations and was assumed to be well mixed with the other reactants. Another condition specified for the C-P case is that the ratios of $A, B$, and $C$ are such that either $A$ or $C$ could consume all of the available $B$, so $B$ is always the limiting reagent.

Simulations for both reaction schemes were run until the equivalent of $t^{*}=500$ in the case of $\mathrm{Da}=1$. Since the simulations are solved in time, the dimensionless times to which the simulations were run were scaled according to the Damköhler number. Therefore, $t^{*}=500$ for $\mathrm{Da}=1$ is equal to $t^{*}=50000$ for $\mathrm{Da}=0.01$ and $t^{*}=0.05$ for $\mathrm{Da}=$ 10000 , that is, the values of $\mathrm{Da} \cdot t^{*}$ are equal for all cases. In fact, $\mathrm{Da} \cdot t^{*}$ is equivalent to a dimensionless reaction time where $\mathrm{Da} \cdot t^{*}=t / \tau_{R}$. Therefore, running the simulations to $\mathrm{Da} \cdot t^{*}=500$ is the same as the simulations being run for 500 reaction times. All these dimensionless times are in fact equal in real time. For most of the cases, it was seen that all of the limiting reagent $B$ is consumed by $t^{*}=500$ or equivalent. COMSOL returned profiles of mass fraction for the various species over the dimensionless space $x^{*}$ for each dimensionless time step $t^{*}$.

\section{Results and Discussion}

The results and discussion are presented as follows. First, the time evolution of species profiles across individual striations is presented and discussed for the competitive-consecutive and competitive-parallel reactions. This discussion includes the effects that mixing and reaction rate ratio have on the species profiles. After that, the definition of yield of desired product is presented, and results showing the time evolution of the yield of desired product for both reaction schemes are shown. This is followed by a discussion of the effects of mixing and reaction rate ratio on the yield of desired product for both reaction schemes.

5.1. Competitive-Consecutive (C-C) Reaction. In order to test the model for the $\mathrm{C}-\mathrm{C}$ reaction, values were assigned to $\mathrm{Da}$ and $k_{2} / k_{1}$ as given in Table 5 . The stoichiometric coefficients were all set to 1 in order to match the classic reaction scheme used by Cox and others (Muzzio and Liu [16], Clifford et al. [6, 19-21], Clifford [17], Clifford and Cox [18], and Cox [7]). This allows comparison of results for the effect of 
TABle 5: Numerical values for simulated C-C test cases. Stoichiometric coefficients $\alpha, \beta, \gamma$, and $\epsilon$ were set to 1 , representing the reaction: $A+B \stackrel{k_{1}^{\prime}}{\rightarrow} P, P+B \stackrel{k_{2}^{\prime}}{\rightarrow} S$, and the initial mass fraction of species $B$ was always $1\left(w_{B_{0}}=1\right)$.

\begin{tabular}{lcc}
\hline C-C case & $k_{2} / k_{1}=(1 / 2)\left(k_{2}^{\prime} / k_{1}^{\prime}\right)$ & $\mathrm{Da}=k_{1}^{\prime}\left(\rho_{T} / M\right)\left(L_{B}^{2} / D_{B}\right)$ \\
\hline 1 & $10^{-5}$ & 1 \\
2 & $10^{-5}$ & 10000 \\
3 & 1 & 1 \\
4 & 1 & 10000 \\
\hline
\end{tabular}

striation thickness and reaction rates with their data. The initial conditions were chosen such that only pure $A$ and $B$ are present in the system.

Looking at the C-C cases in Table 5 , the values of $k_{2} / k_{1}$ and $\mathrm{Da}$ for Case 1 are favourable conditions for a high yield of $P$, that is, $k_{2} / k_{1} \ll 1$ and $\mathrm{Da}=1$. For Case 4 , the yield of $P$ should be small, that is, $k_{2} / k_{1}=1$ and $\mathrm{Da} \gg 1$. The two cases are meant to represent the two extremes of very favorable reaction rate ratio and perfect mixing and very unfavorable kinetics and poor mixing conditions. Cases 2 and 3 have good $k_{2} / k_{1}$ with bad mixing and bad $k_{2} / k_{1}$ with good mixing. The solutions COMSOL returns are the profiles of mass fraction for the various species over the dimensionless space $x^{*}$ for each time step $t^{*}$. Figure 2 shows the spatial and temporal evolution of species over a single dimensionless striation. Before discussing the profiles in detail, it is important to note a couple of points about the profiles. A vertical line represents a sharp interface. A curved line represents a gradient in the concentration. Finally, a horizontal line represents uniform concentration across the space.

Looking at Figures 2(a) and 2(c), one can see that all of the species are uniformly distributed for all time steps greater than $t^{*}=0$. This is not the case for Figures 2(b) and 2(d). This can be attributed to the smaller striation thicknesses, that is, the lower Damköhler number, for Cases 1 and 3. As the striations are thinner for those cases, the species can completely diffuse across in a shorter amount of time than for Cases 2 and 4 where the striations are 100 times thicker. The thicker striations allow for spatial inhomogeneity of the species. The thinner striation thicknesses allow for differences only in temporal distribution of species and not spatial distribution. The thicker striations cause differences in both temporal and spatial distributions. Figures 2(b) and 2(d) also exhibit an interface between reactants whereas Figures 2(a) and 2(c) do not.

Despite the fact that there is complete mixing for both Cases 1 and 3, there is a very large difference in the yield of $P$ for the two cases. For Case 1, which has both good mixing and a favourable reaction rate ratio, the majority of the mass present is that of $P$, the desired product (Figure 2(a)-(iii)). For Case 3, however, the mass fraction of the undesired product is always higher than that of the desired product (Figure 2(c)-(iii)). There is a significant drop in mass fraction of $P$ from 0.99 to 0.25 , showing the dramatic effect of reaction rate ratio for the same mixing conditions.
Looking at Figures 2(b)-(iii) and 2(d)-(iii), the same reversal of $P$ and $S$ is observed. The reaction rate ratio has a profound effect on the yield of desired product that is independent of mixing. When the reaction rate ratio is good, the undesired reaction does not participate. All of the product $P$ forms at the interface of $A$ and $\mathrm{B}$, making the profile of mass fraction of $P$ symmetric about the mid-plane, $x^{*}=$ 0 , as shown in Figures 2(b)-(ii) and 2(b)-(iii). When the undesirable by-product reaction occurs at a comparable rate to that of the desired reaction, a significant asymmetry in the profiles of mass fraction for all species is visible (Figures 2(d)(ii) and 2(d)-(iii)). This can be attributed to the fact that the second reaction occurs only on the right hand side of $x^{*}=0$ where $P$ is in contact with $B$. This causes $P$ to react to form $S$ when it is exposed to $B$ on one side.

The key results are as follows: first, a small striation thickness allows for uniform concentrations of species, that is, perfect mixing, whereas larger striations can cause spatial inhomogeneities in species mass fraction. Second, the reaction rate ratio is an independent factor which can significantly alter the yield of desired product regardless of the mixing condition. This effect is predictable in the sense that if the ratio is good the yield is good, and if the ratio is poor the yield is poor. Finally, for the larger striation thicknesses, a good reaction ratio causes symmetric concentration profiles of desired product $P$, while a bad ratio causes the product profiles to skew towards the $B$ side of the striation. Perfect mixing simplifies the reaction analysis and shortens the reaction time. Having favourable kinetics improves the yield significantly.

Changing the stoichiometry did not affect the species profiles in the case of good reaction rate ratio $\left(k_{2} / k_{1}=\right.$ $10^{-5}$ ) for both good and bad mixing conditions $(1<\mathrm{Da}<$ 10000). The profiles of all the species were identical to those presented above. The species profiles for the different stoichiometries with the bad reaction rate ratio $\left(k_{2} / k_{1}=1\right)$ and good mixing $(\mathrm{Da}=1)$ look similar to those shown here, but the amount of $P$ and $S$ produced changes. The case of both bad rate ratio $\left(k_{2} / k_{1}=1\right)$ and poor mixing $(\mathrm{Da}=$ 10000 ) always resulted in a larger amount of $S$ produced than $P$, and all the profiles were skewed towards $B$. The amount of $S$ and $P$ produced varies with stoichiometry, and the profiles are skewed more or less depending on the stoichiometry.

5.2. Competitive-Parallel (C-P) Reaction. Table 6 shows the variable settings for the $\mathrm{C}-\mathrm{P}$ simulations. The four cases are identical to the ones used for the $\mathrm{C}-\mathrm{C}$ simulations with the exception of the definition of $k_{2} / k_{1}$. The stoichiometry illustrated is the classic C-P reaction scheme with all stoichiometric coefficients set to 1 . The initial conditions were chosen such that $w_{B_{0}}=0.5$, and the initial amounts of $A, C$, and $I$ present in the system were calculated using the formulae in Table 4. The solutions COMSOL returns are the profiles of mass fraction for the various species over the dimensionless space $x^{*}$ for each time step $t^{*}$. Figure 3 shows the spatial and temporal evolution of species including the inert.

The C-P case profiles show many of the same characteristics as the $\mathrm{C}-\mathrm{C}$ cases. Cases 1 and 3, with the thinner initial striations, once again show spatial homogeneity for 

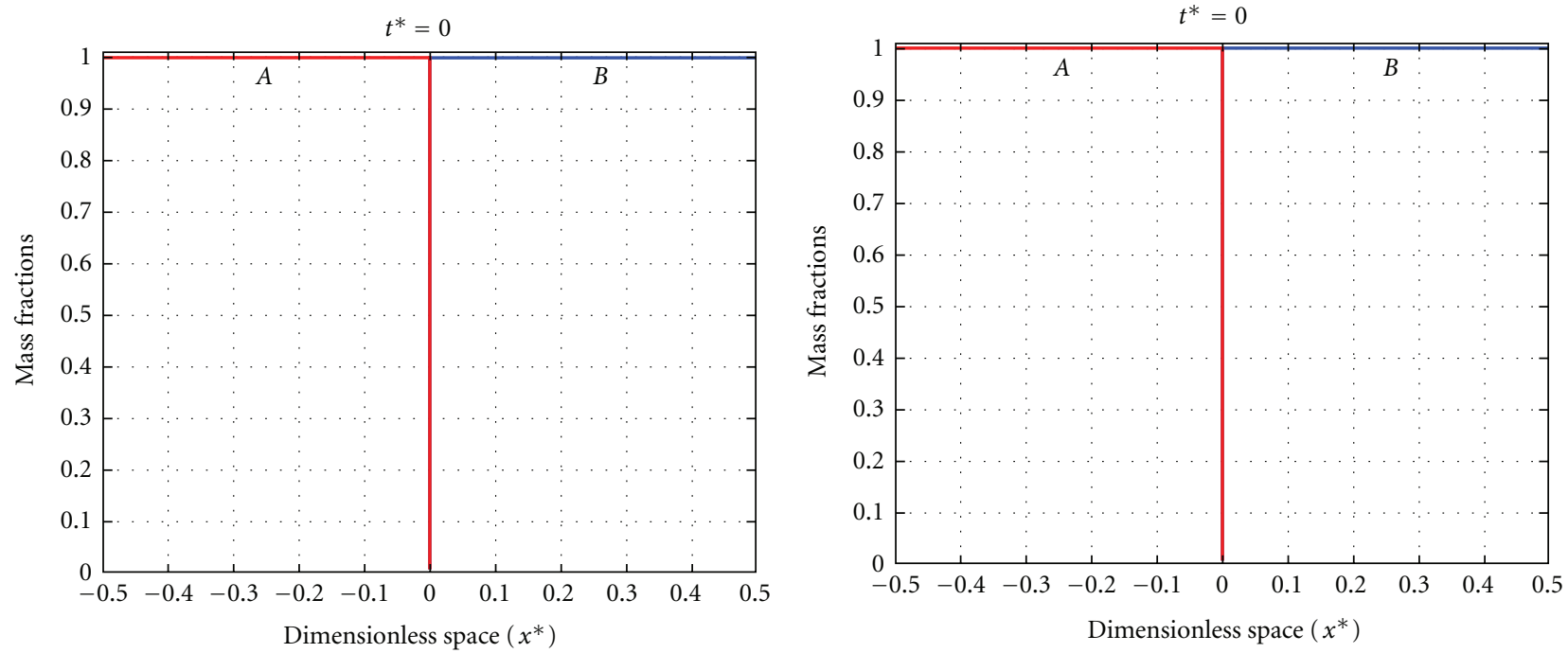

(i) $\mathrm{Da} \cdot t^{*}=0$

(i) $\mathrm{Da} \cdot t^{*}=0$
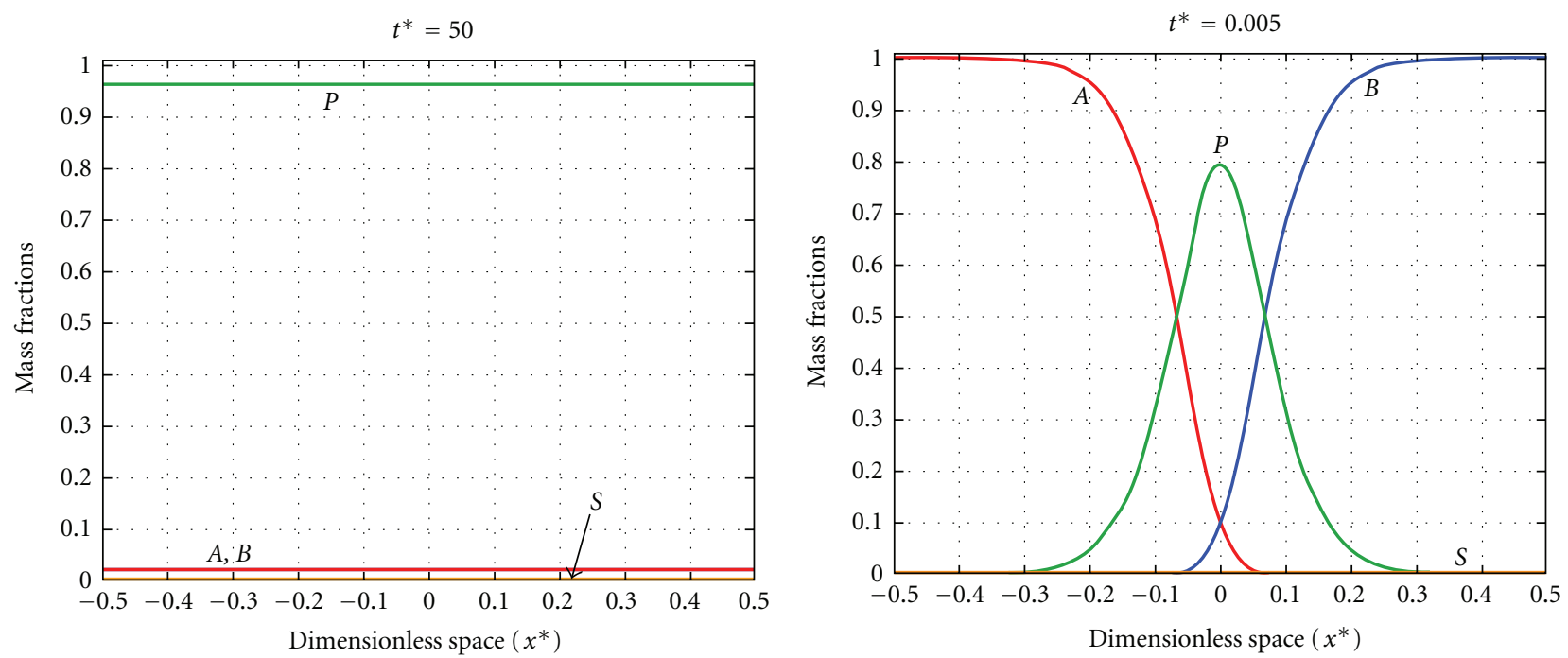

(ii) $\mathrm{Da} \cdot t^{*}=50$

$t^{*}=500$

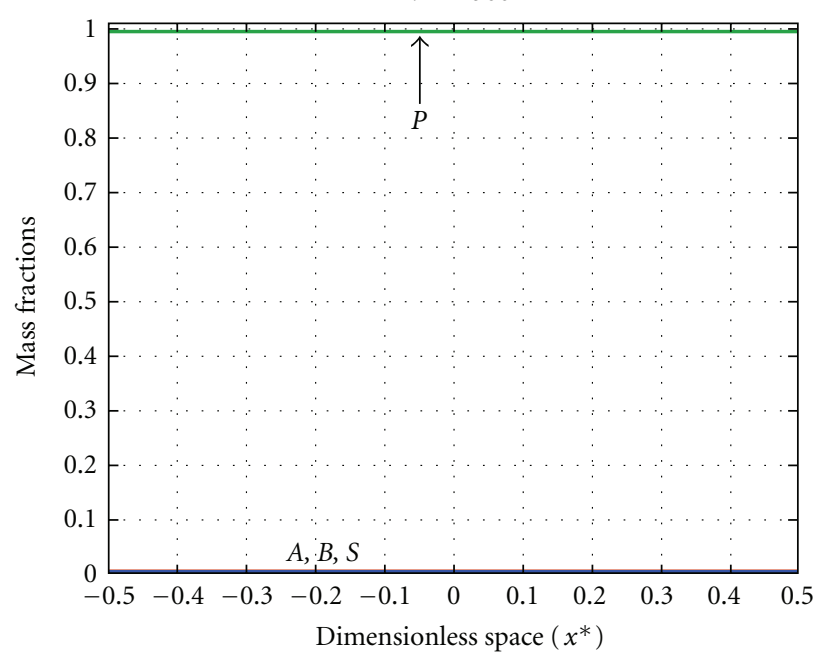

(iii) $\mathrm{Da} \cdot t^{*}=500$

(a) Case $1, k_{2} / k_{1}=10^{-5}, \mathrm{Da}=1$

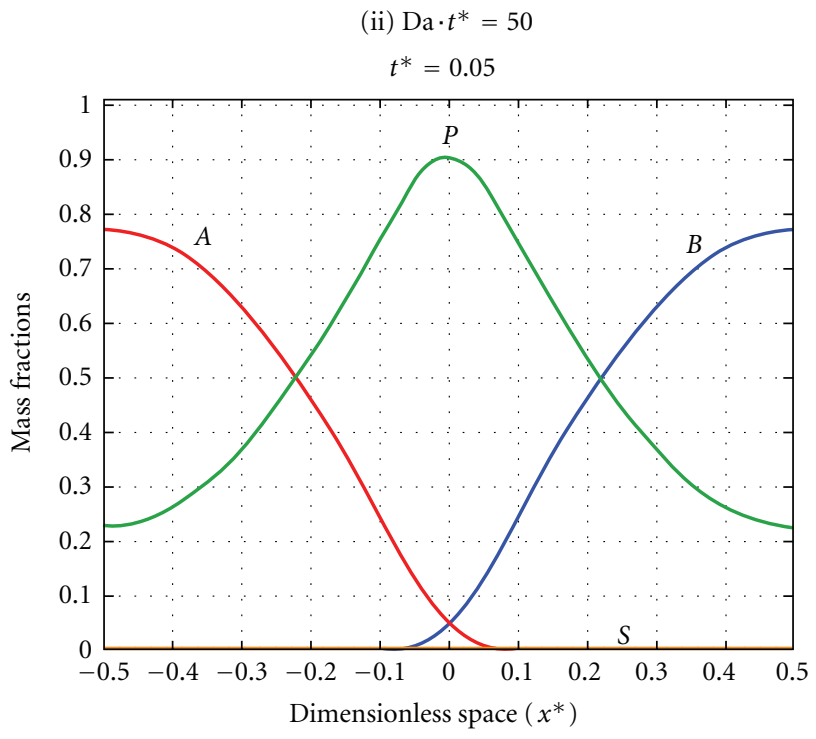

(iii) $\mathrm{Da} \cdot t^{*}=500$

(b) Case $2, k_{2} / k_{1}=10^{-5}, \mathrm{Da}=10000$

Figure 2: Continued. 

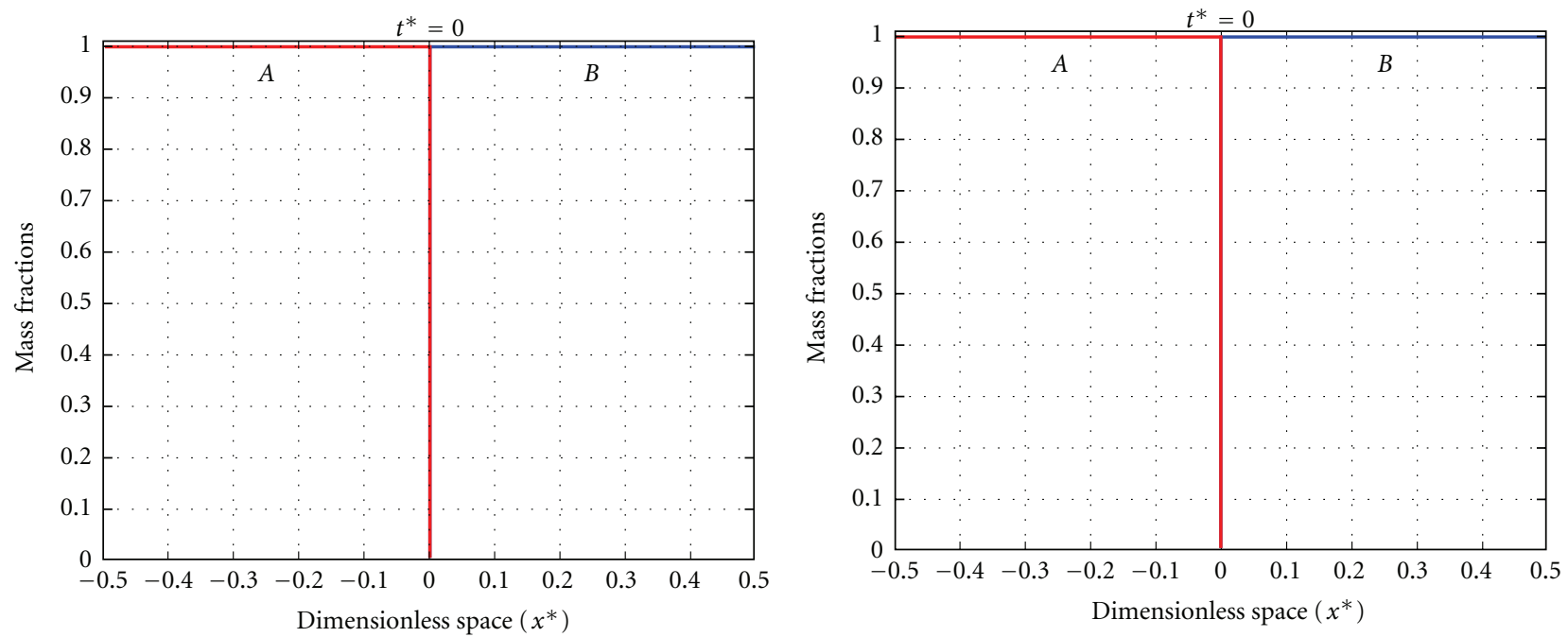

(i) $\mathrm{Da} \cdot t^{*}=0$

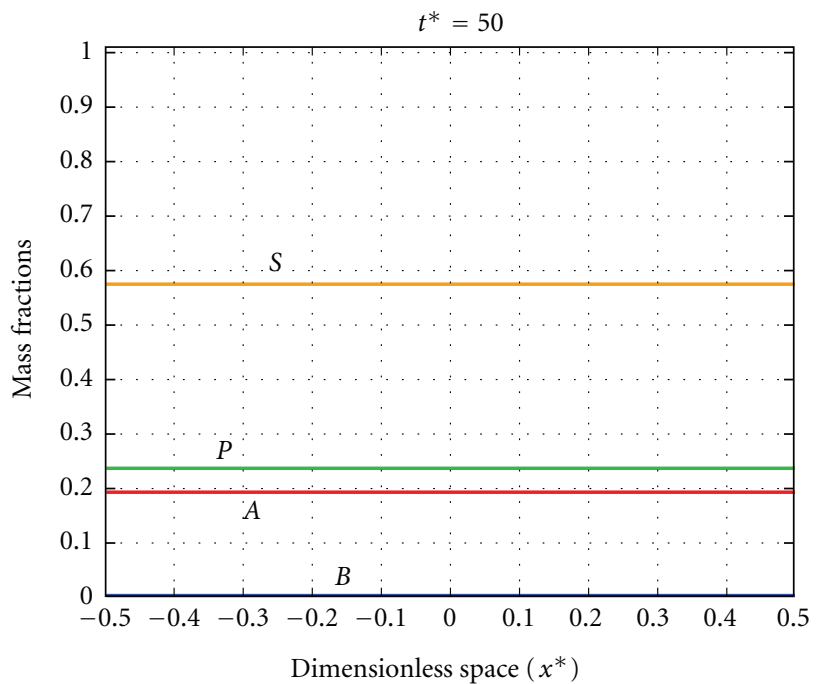

(i) $\mathrm{Da} \cdot t^{*}=0$

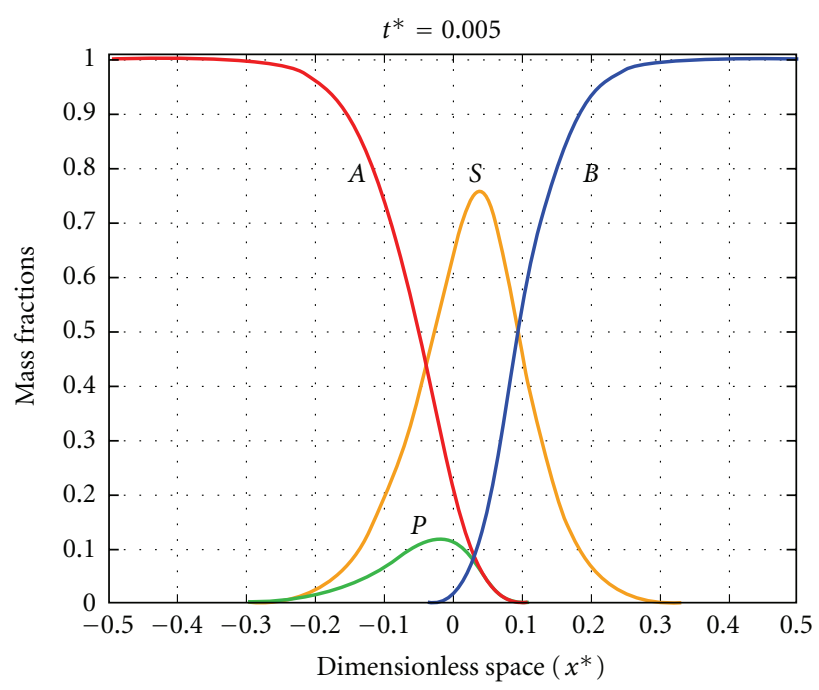

(ii) $\mathrm{Da} \cdot t^{*}=50$

(ii) $\mathrm{Da} \cdot t^{*}=50$

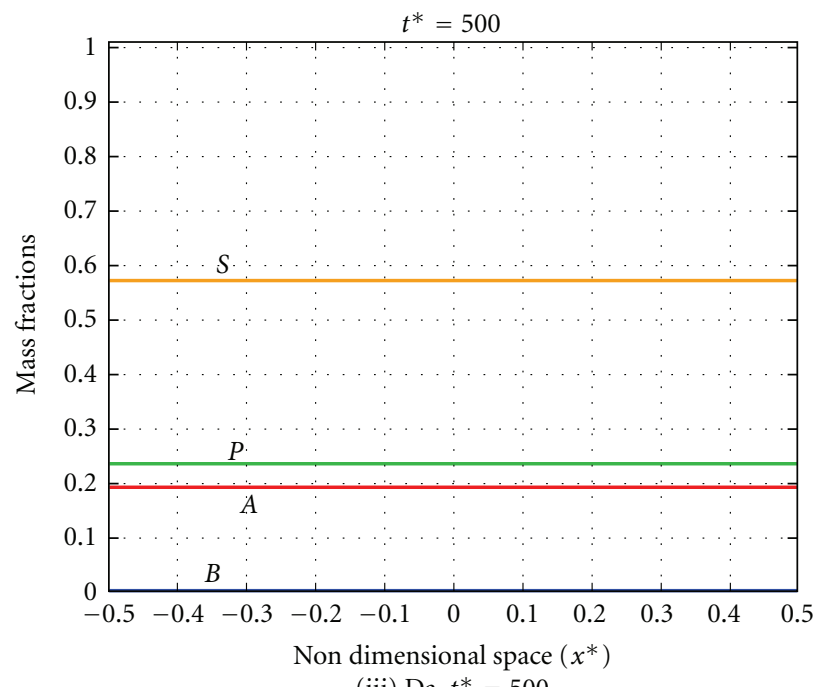

(iii) $\mathrm{Da} \cdot t^{*}=500$

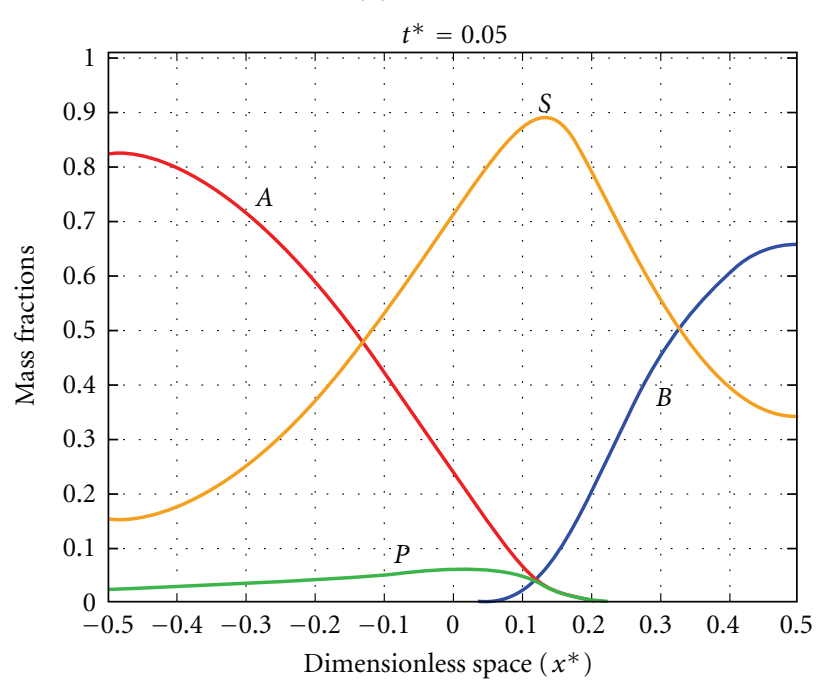

(iii) $\mathrm{Da} \cdot t^{*}=500$

(c) Case $3, k_{2} / k_{1}=1, \mathrm{Da}=1$

(d) Case $4, k_{2} / k_{1}=1, \mathrm{Da}=10000$

FIgURE 2: Spatial and temporal evolution of mass fractions for C-C cases (a) Case $1\left(k_{2} / k_{1}=10^{-5}, \mathrm{Da}=1\right)$; (b) Case $2\left(k_{2} / k_{1}=10^{-5}, \mathrm{Da}=\right.$ $10000)$; (c) Case $3\left(k_{2} / k_{1}=1, \mathrm{Da}=1\right)$; and (d) Case $4\left(k_{2} / k_{1}=1, \mathrm{Da}=10000\right)$. 


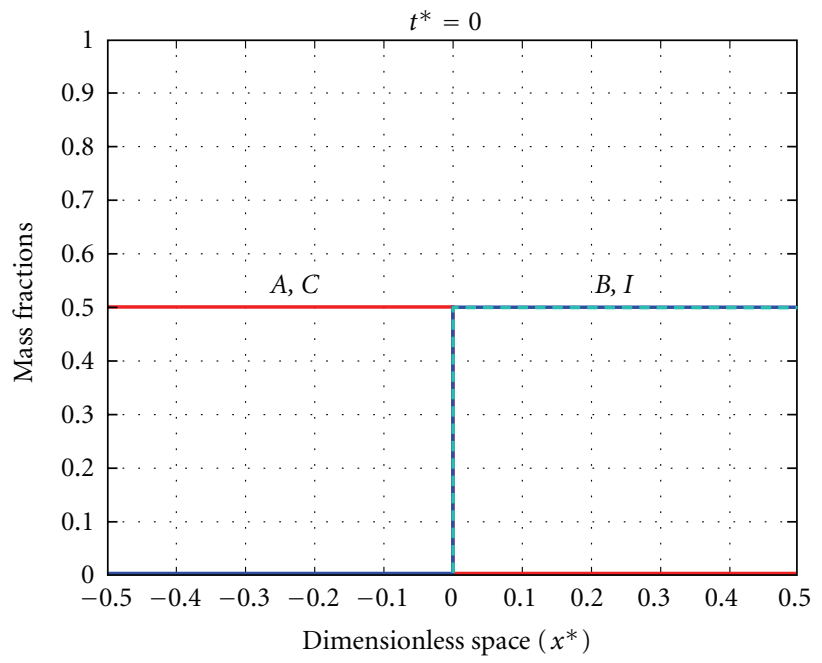

(i) $\mathrm{Da} \cdot t^{*}=0$

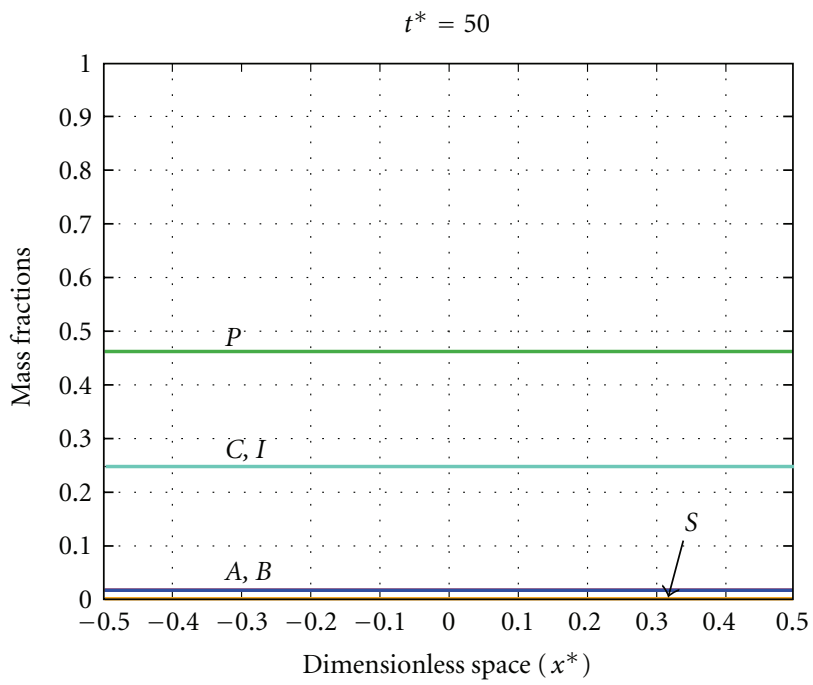

(ii) $\mathrm{Da} \cdot t^{*}=50$

$$
t^{*}=500
$$

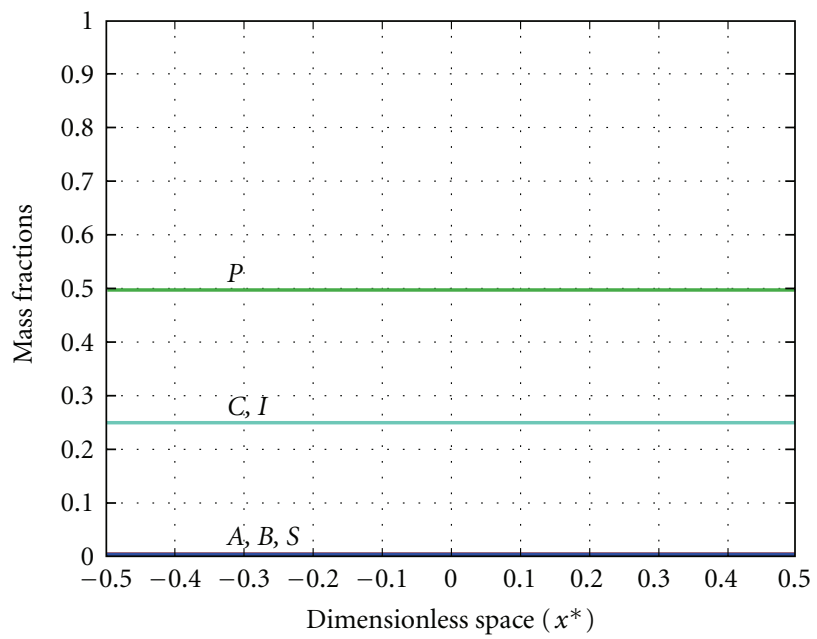

(iii) $\mathrm{Da} \cdot t^{*}=500$

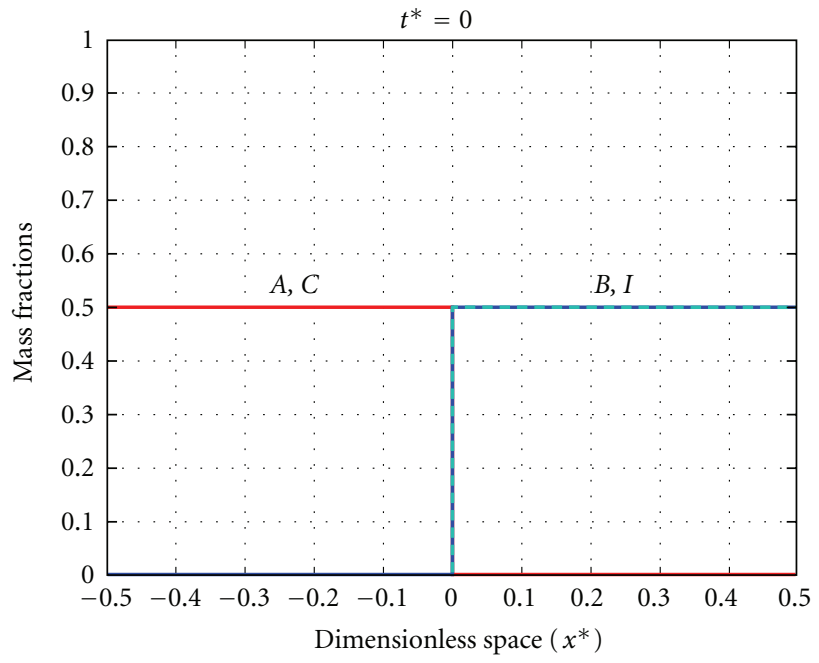

(i) $\mathrm{Da} \cdot t^{*}=0$

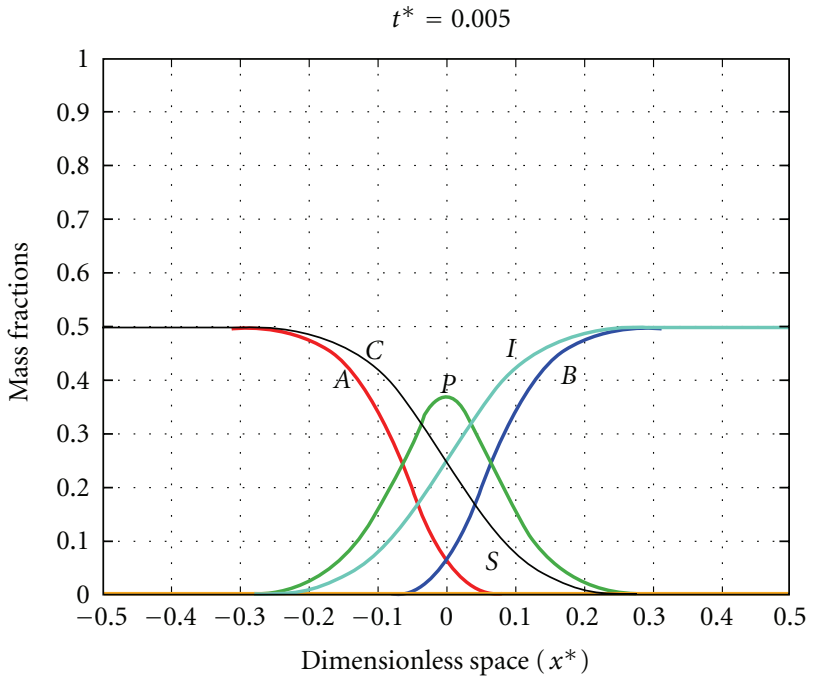

(ii) $\mathrm{Da} \cdot t^{*}=50$

$t^{*}=0.05$

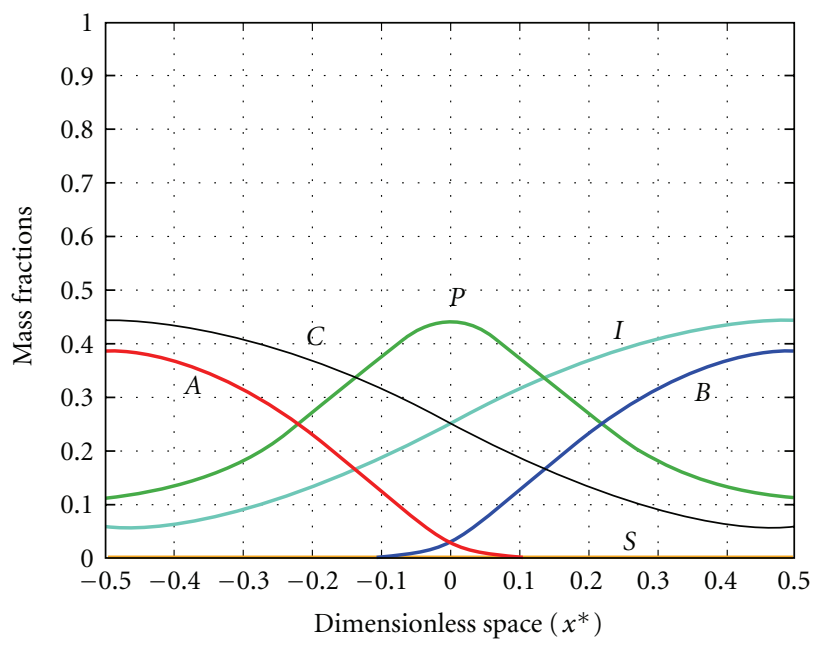

(iii) $\mathrm{Da} \cdot t^{*}=500$

(b) $k_{2} / k_{1}=10^{-5}, \mathrm{Da}=10000$

Figure 3: Continued. 

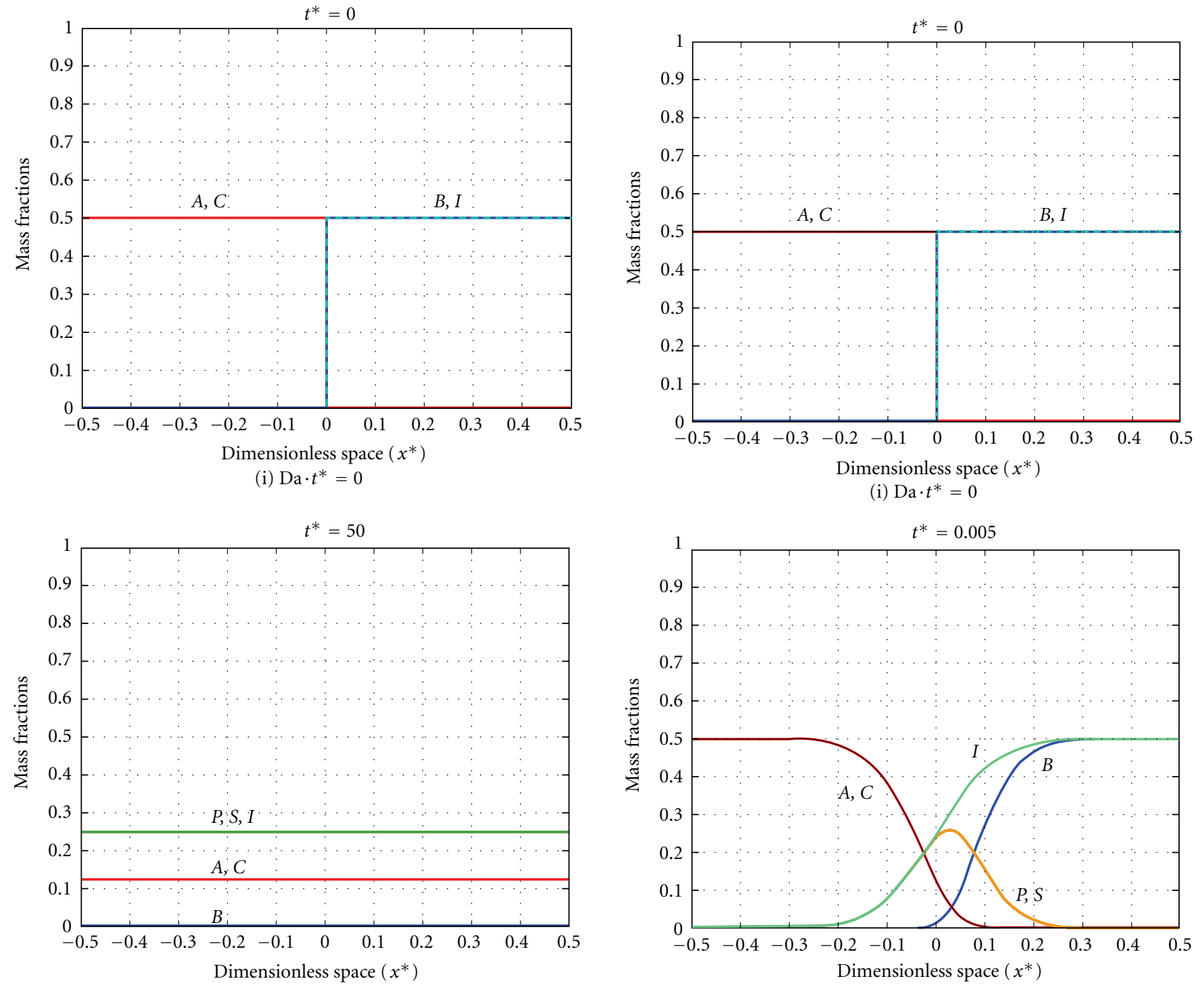

(ii) $\mathrm{Da} \cdot t^{*}=50$

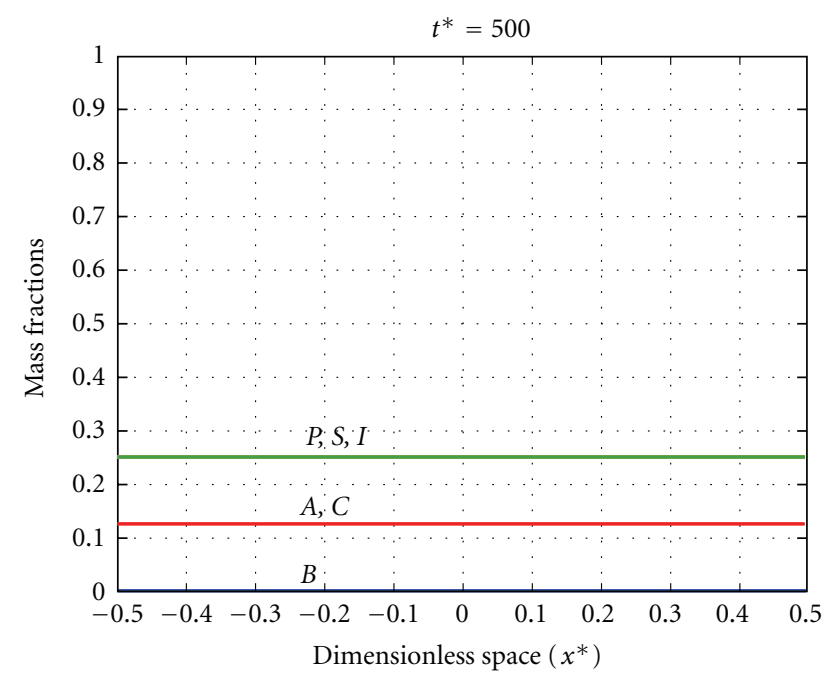

(iii) $\mathrm{Da} \cdot t^{*}=500$

(ii) $\mathrm{Da} \cdot t^{*}=50$

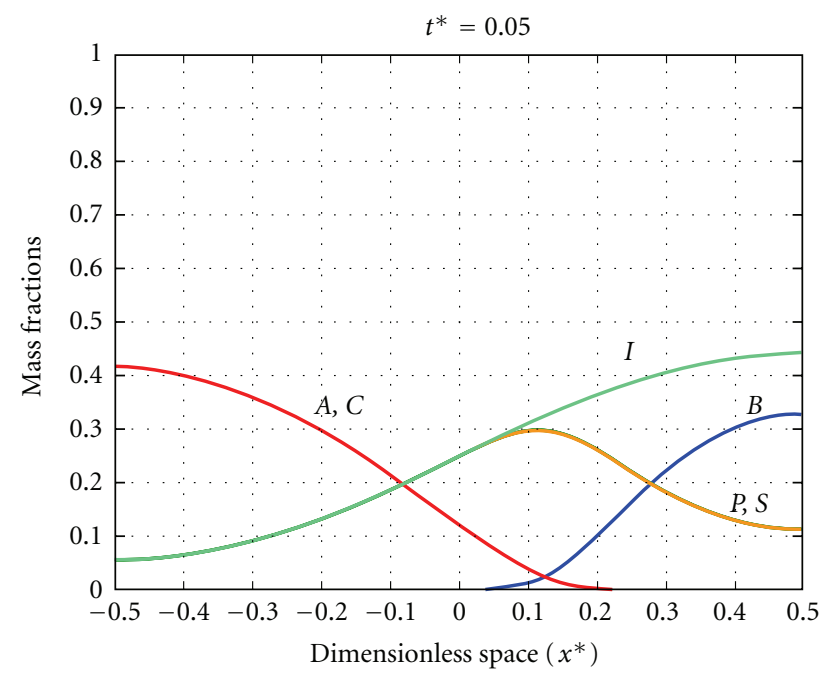

(iii) $\mathrm{Da} \cdot t^{*}=500$

(c) $k_{2} / k_{1}=1, \mathrm{Da}=1$

(d) $k_{2} / k_{1}=1, \mathrm{Da}=10000$

FIGURE 3: Spatial and temporal evolution of mass fractions for C-P cases (a) Case $1\left(k_{2} / k_{1}=10^{-5}, \mathrm{Da}=1\right)$; (b) Case $2\left(k_{2} / k_{1}=10^{-5}, \mathrm{Da}=\right.$ $10000)$; (c) Case $3\left(k_{2} / k_{1}=1, \mathrm{Da}=1\right)$; and (d) Case $4\left(k_{2} / k_{1}=1, \mathrm{Da}=10000\right)$. 
TABle 6: Numerical values for simulated C-P test cases. Stoichiometric coefficients $\gamma$ and $\epsilon$ were set to 1 , representing the reaction: $A+B \stackrel{k_{1}^{\prime}}{\rightarrow} P, C+B \stackrel{k_{2}^{\prime}}{\rightarrow} S$, and the initial mass fraction of species $B$ was always $0.5\left(w_{B_{0}}=0.5\right)$.

\begin{tabular}{lcc}
\hline $\mathrm{C}-\mathrm{P}$ case & $k_{2} / k_{1}=k_{2}^{\prime} / k_{1}^{\prime}$ & $\mathrm{Da}=k_{1}^{\prime}\left(\rho_{T} / M\right)\left(L_{B}^{2} / D_{B}\right)$ \\
\hline 1 & $10^{-5}$ & 1 \\
2 & $10^{-5}$ & 10000 \\
3 & 1 & 1 \\
4 & 1 & 10000 \\
\hline
\end{tabular}

all the species across the entire striation thickness, whereas Cases 2 and 4 show spatial variations in mass fraction for all the species across the striations. The yields of $P$ and $S$ change when the reaction ratio is varied from favourable $\left(k_{2} / k_{1}=\right.$ $\left.10^{-5}\right)$ to unfavourable $\left(k_{2} / k_{1}=1\right)$. Cases 1 and 2 have a high yield of $P$; Cases 3 and 4 have an equal yield of $P$ and $S$. The cases with the large striation thicknesses $(\mathrm{Da}=10000)$ show symmetry in product mass fraction profiles about $x^{*}=0$ when the second reaction is insignificant $\left(k_{2} / k_{1}=10^{-5}\right)$ and significant asymmetry when it is actively participating in the reaction $\left(k_{2} / k_{1}=1\right)$. The main difference between the $\mathrm{C}-\mathrm{C}$ and $\mathrm{C}-\mathrm{P}$ reactions is that once the product $P$ is formed in the $\mathrm{C}-\mathrm{P}$ reaction, it does not get consumed by a side reaction. Therefore, in terms of measuring the yield of $P$, the C-P reaction scheme is a lot less mixing sensitive than the $\mathrm{C}-\mathrm{C}$ reaction scheme. For the $\mathrm{C}-\mathrm{C}$ reaction, the longer that $P$ sits in contact with $B$, the higher the chance that the yield of $P$ will decrease.

Changing the stoichiometry for the C-P reactions resulted in some non-linear profile changes. While the profiles for the well-mixed cases remained uniform across the striation, the magnitudes of desired and undesired product produced changed. The profiles for the poorly mixed cases look different from the profiles presented here owing partly to the different initial conditions required when the stoichiometry was changed and also because of the stoichiometries themselves. In a second paper [29], the yield of $P$ is used to capture all of these changes for both the $\mathrm{C}-\mathrm{P}$ as well as the $\mathrm{C}-\mathrm{C}$ reaction schemes.

5.3. Yield of Desired Product P. In order to to assess yield for the non-uniform profiles of mass fraction, the profiles of $P$ were integrated to obtain the total mass of $P$ present in the system at an instant in time:

$$
Y_{P}=\frac{\text { mass of species } P \text { at } t^{*}}{\text { max mass of } P \text { obtainable }}=\frac{\int_{-0.5}^{0.5} w_{P} d x^{*}\left(t^{*}\right)}{0.5 w_{B_{0}}(1+(1 / \epsilon))}
$$

Following $Y_{P}$ over time gives the progression of yield over time. Figure 4 shows the yield of $P$ over time as the reaction progresses for the four $\mathrm{C}-\mathrm{C}$ cases, and Figure 5 shows the same results for the four C-P cases. Plotting $\mathrm{Da} \cdot t^{*}$ on the $x$ axis allows all four curves to be displayed on the same figure. These figures confirm the conclusions drawn above that good mixing and a good reaction rate ratio will maximize the yield of desired product (Case 1 in both Figures 4 and 5 ), and poor mixing with an unfavourable reaction rate

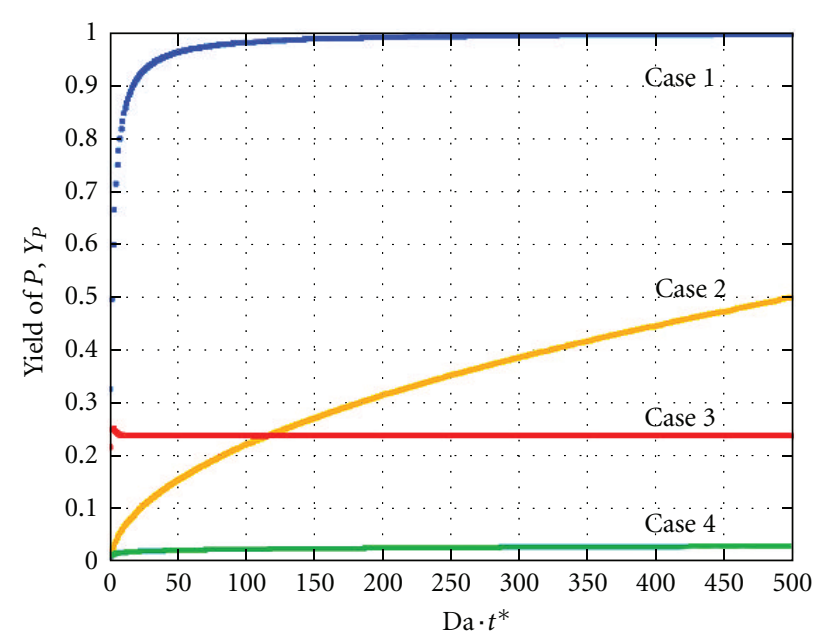

Figure 4: Yield of $P$ versus time for the C-C cases.

ratio minimizes the yield of desired product (Case 4 for both Figures 4 and 5). This also confirms the notion that minimization of the Damköhler number and reaction rate ratio is desirable. Clifford et al.s [6] conclusions for the classic C-C stoichiometry are the same as ours. Quantitative comparison is not possible because our characteristic length and time scale choices are different from theirs.

5.4. Effect of Reaction Rate Ratio on Yield of P. Figures 4 and 5 show that the reaction rate ratio affects how much product is formed regardless of the mixing condition. This is evident in the comparison of Cases 1 and 3, which are both well mixed, and Cases 2 and 4, which are both poorly mixed. The favourable reaction rate ratio (Cases 1 and 2) always provides a higher final yield of $P$. It is impossible to get a good yield of desired product if the reaction rate ratio is unfavourable, regardless of the mixing condition (Cases 3 and 4). Clifford et al. [6] came to the same conclusion for the effect of reaction rate ratio. A smaller reaction rate ratio is favourable for maximizing the yield of desired product.

5.5. Effect of Mixing on Yield of $P$. There is a significant effect of mixing on $Y_{P}$ evident in Figures 4 and 5. When the reaction rate ratio is favourable, having good mixing can cause a substantial increase in yield of desired product, as seen by comparing Cases 1 and 2 in both the figures $\left(Y_{P}=\right.$ 0.5 to 1 for $\mathrm{C}-\mathrm{C}$ and $Y_{P}=0.5$ to 1 for $\mathrm{C}-\mathrm{P}$ ). If the reaction rate ratio is unfavourable, a similar favourable effect of mixing is seen by comparing Cases 3 and 4, though it is not as profound as when the reaction rate ratio is good $\left(Y_{P}=0.03\right.$ to 0.24 for C-C and $Y_{P}=0.33$ to 0.5 for $\mathrm{C}-\mathrm{P}$ ). This illustrates that the effect of mixing is limited by the reaction rate ratio, that is, the reaction rate ratio determines the final yield and good mixing helps one to realise that asymptotic value of yield. For the well-mixed cases (Cases 1 and 3 ) the final yield of $P$ is attained much faster, so mixing also determines the pace of the reaction. In the $\mathrm{C}-\mathrm{C}$ case, due to the nature 


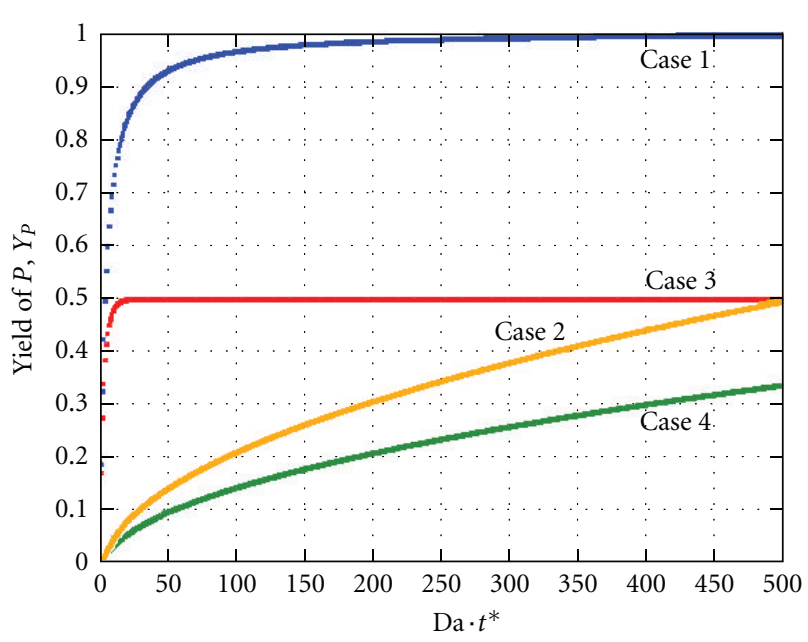

Figure 5: Yield of $P$ versus time for the C-P cases.

of the reaction, this has a significant positive effect on the final yield of $P$. Cox and others came to a similar conclusion (Cox et al. [5] and Clifford et al. [6]): minimizing the Damköhler number leads to an increase in the yield of desired product.

\section{Conclusions}

New definitions of Damköhler number (Da) and dimensionless reaction rate ratio $\left(k_{2} / k_{1}\right)$ were derived to investigate the effects of stoichiometry, mixing, and reaction rate ratio for competitive-consecutive and competitive-parallel reactions with a general stoichiometry. The model development deals with both reaction schemes, something that was not previously available. A single Damköhler number was found for both kinds of mixing sensitive reactions. This is encouraging, since there has previously been a lot of debate on the formulation of the Damköhler number for these competing reactions, for example, whether it should be based on the first or the second reaction. Having just one expression to describe mixing is more physical, since mixing should be independent of the reaction scheme. The general expression for the Damköhler number, when applied to the classic reaction schemes, collapses to the expressions used in previous investigations, Cox et al. [5] in particular. The C-C and C-P reaction schemes, however, are very different and these differences are reflected in the specific reaction rate ratios for the two types of reaction stoichiometries that are to be used in the model.

The effects of mixing and reaction rate ratios on the yield of desired product were investigated for the classic competitive-consecutive and competitive-parallel reaction schemes. The reaction rate ratio ultimately limits the final yield of desired product. Mixing determines whether that yield is achieved, and the rate at which the final yield is reached. The reaction rate ratio and the Damköhler number both need to be minimized to achieve the maximum yield of product. The results from the model agree with the expected results and with the results of previous investigations where both mixing and reaction rate ratio were varied. Improving the mixing and chemistry by minimizing the Damköhler number and the reaction rate ratio is desirable and leads to improvements in yield of desired product.

This confirmation of the model allows for future work where the effects of stoichiometry, mixing and $k_{2} / k_{1}$ ratios on yield will be investigated for a general mixing sensitive reaction with varying stoichiometry.

While the model allows for the investigation of the effects of initial concentrations of reactants, this was not done in the current study and this is another possible avenue for further exploration. It is also acknowledged that the $1 \mathrm{D}$ model is not an accurate depiction of real turbulent mixing, but this investigation was meant to be a first foray into the effect of stoichiometry of mixing sensitive reactions, so simplicity was desirable. Added complexities include introducing fluid flow into the model, such as taking into account laminar stretching of the striations, 2D deformation and eventually $3 \mathrm{D}$ deformation, similar to the works of Cox [7] and Baldyga and Bourne [1]. The eventual goal would be to integrate the reaction diffusion equations into the Engulfment model of Baldyga and Bourne [1]. This model could also be integrated into $3 \mathrm{D}$ meso-mixing models to better approximate the real industrial situation.

\section{Nomenclature}

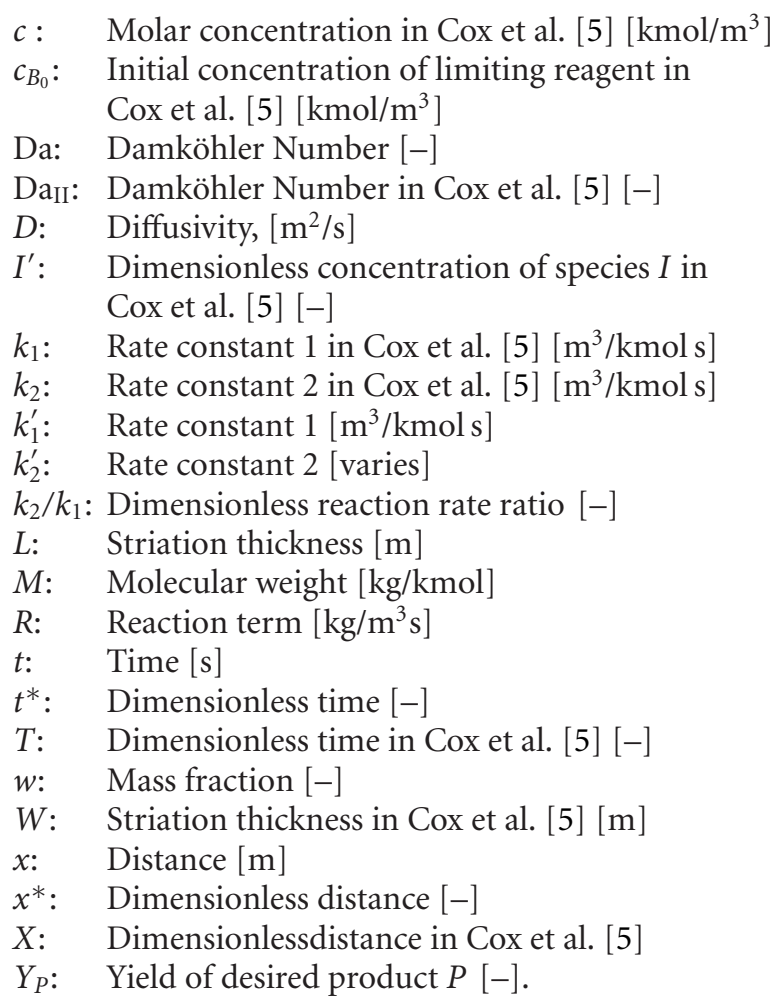

\section{Greek Letters}
$\alpha, \beta, \gamma, \epsilon:$ Stoichiometric Coefficients [-]
$\varepsilon: \quad$ Reaction rate ratio in Cox et al. [5] [-]
$\rho: \quad$ Mass concentration $\left[\mathrm{kg} / \mathrm{m}^{3}\right]$ 
$\tau_{M}:$ Mixing time $[\mathrm{s}]$

$\tau_{R}:$ Reaction time $[\mathrm{s}]$.

\section{Subscripts}

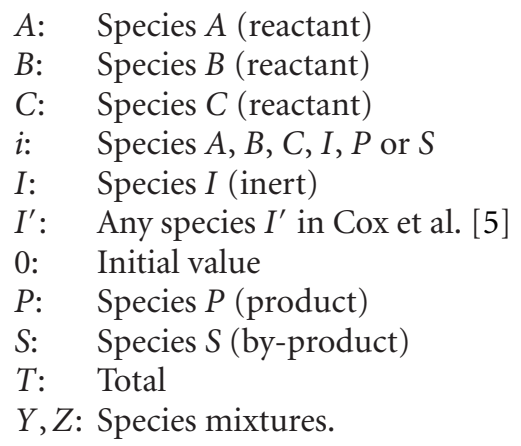

\section{Acknowledgments}

The authors would like to thank NSERC Canada and MITACS for funding provided to carry out this research.

\section{References}

[1] J. Baldyga and J. R. Bourne, Turbulent Mixing and Chemical Reactions, Wiley, Chichester, UK, 1999.

[2] G. K. Patterson, E. L. Paul, S. M. Kresta, and A. W. Etchells III, "Mixing and chemical reactions," in Handbook of Industrial Mixing-Science and Practice, E. L. Paul, V. A. Atiemo-Obeng, and S. M. Kresta, Eds., Wiley, Hoboken, NJ, USA, 2004.

[3] J. Baldyga and J. R. Bourne, "Interactions between mixing on various scales in stirred tank reactors," Chemical Engineering Science, vol. 47, no. 8, pp. 1839-1848, 1992.

[4] J. Badyga, J. R. Bourne, and S. J. Hearn, "Interaction between chemical reactions and mixing on various scales," Chemical Engineering Science, vol. 52, no. 4, pp. 457-466, 1997.

[5] S. M. Cox, M. J. Clifford, and E. P. L. Roberts, "A two-stage reaction with initially separated reactants," Physica A, vol. 256, no. 1-2, pp. 65-86, 1998.

[6] M. J. Clifford, E. P. L. Roberts, and S. M. Cox, "The influence of segregation on the yield for a series-parallel reaction," Chemical Engineering Science, vol. 53, no. 10, pp. 1791-1801, 1998.

[7] S. M. Cox, "Chaotic mixing of a competitive-consecutive reaction," Physica D, vol. 199, no. 3-4, pp. 369-386, 2004.

[8] P. V. Danckwerts, "The definition and measurement of some characteristics of mixtures," Applied Scientific Research, Section A, vol. 3, no. 4, pp. 279-296, 1952.

[9] P. V. Danckwerts, "The effect of incomplete mixing on homogeneous reactions," Chemical Engineering Science, vol. 8, no. 1-2, pp. 93-102, 1958.

[10] O. Levenspiel, Chemical Reaction Engineering, Wiley, New York, NY, USA, 2nd edition, 1972.

[11] S. Bhattacharya, Performance improvement of stirred tank reactors with surface feed [Ph.D. thesis], University of Alberta, Edmonton, Canada, 2005.

[12] J. Baldyga and R. Pohorecki, "Turbulent micromixing in chemical reactors-a review," The Chemical Engineering Journal and The Biochemical Engineering Journal, vol. 58, no. 2, pp. 183-195, 1995.

[13] J. Villermaux and L. Falk, "A generalized mixing model for initial contacting of reactive fluids," Chemical Engineering Science, vol. 49, no. 24, pp. 5127-5140, 1994.
[14] R. O. Fox, "On the relationship between Lagrangian micromixing models and computational fluid dynamics," Chemical Engineering and Processing, vol. 37, no. 6, pp. 521-535, 1998.

[15] R. O. Fox, Computational Models for Turbulent Reacting Flows, Cambridge University Press, Cambridge, UK, 2003.

[16] F. J. Muzzio and M. Liu, "Chemical reactions in chaotic flows," Chemical Engineering Journal and the Biochemical Engineering Journal, vol. 64, no. 1, pp. 117-127, 1996.

[17] M. J. Clifford, "A Gaussian model for reaction and diffusion in a lamellar structure," Chemical Engineering Science, vol. 54, no. 3, pp. 303-310, 1999.

[18] M. J. Clifford and S. M. Cox, "A simple model for a twostage chemical reaction with diffusion," IMA Journal of Applied Mathematics, vol. 63, no. 3, pp. 307-318, 1999.

[19] M. J. Clifford, S. M. Cox, and E. P. L. Roberts, "Lamellar modelling of reaction, diffusion and mixing in a two-dimensional flow," Chemical Engineering Journal, vol. 71, no. 1, pp. 49-56, 1998.

[20] M. J. Clifford, S. M. Cox, and E. P. L. Roberts, "Reaction and diffusion in a lamellar structure: the effect of the lamellar arrangement upon yield," Physica A, vol. 262, no. 3-4, pp. 294306, 1999.

[21] M. J. Clifford, S. M. Cox, and E. P. L. Roberts, "The influence of a lamellar structure upon the yield of a chemical reaction," Chemical Engineering Research and Design, vol. 78, no. 3, pp. 371-377, 2000.

[22] I. Hecht and H. Taitelbaum, "Perturbation analysis for competing reactions with initially separated components," Physical Review E, vol. 74, no. 1, Article ID 012101, 2006.

[23] M. Sinder, "Theory for competing reactions with initially separated components," Physical Review E, vol. 65, no. 3, Article ID 037104, pp. 037104/1-037104/4, 2002.

[24] M. Sinder, J. Pelleg, V. Sokolovsky, and V. Meerovich, "Competing reactions with initially separated components in the asymptotic time region," Physical Review E, vol. 68, no. 2, Article ID 022101, 4 pages, 2003.

[25] H. Taitelbaum, B. Vilensky, A. Lin, A. Yen, Y. E. L. Koo, and R. Kopelman, "Competing reactions with initially separated components," Physical Review Letters, vol. 77, no. 8, pp. 1640$1643,1996$.

[26] S. Cornell and M. Droz, "Exotic reaction fronts in the steady state," Physica D, vol. 103, no. 1-4, pp. 348-356, 1997.

[27] S. M. Cox and M. D. Finn, "Behavior of the reaction front between initially segregated species in a two-stage reaction," Physical Review E, vol. 63, no. 5 I, pp. 511021-511027, 2001.

[28] H. S. Fogler, Elements of Chemical Reaction Engineering, Prentice Hall, Upper Saddle River, NJ, USA, 3rd edition, 1999.

[29] S. I. A. Shah, L. W. Kostiuk, and S. M. Kresta, "The effects of mixing, reaction rates and stoichiometry on yield for mixing sensitive reactions, Part II: design protocols," International Journal of Chemical Engineering. In press. 

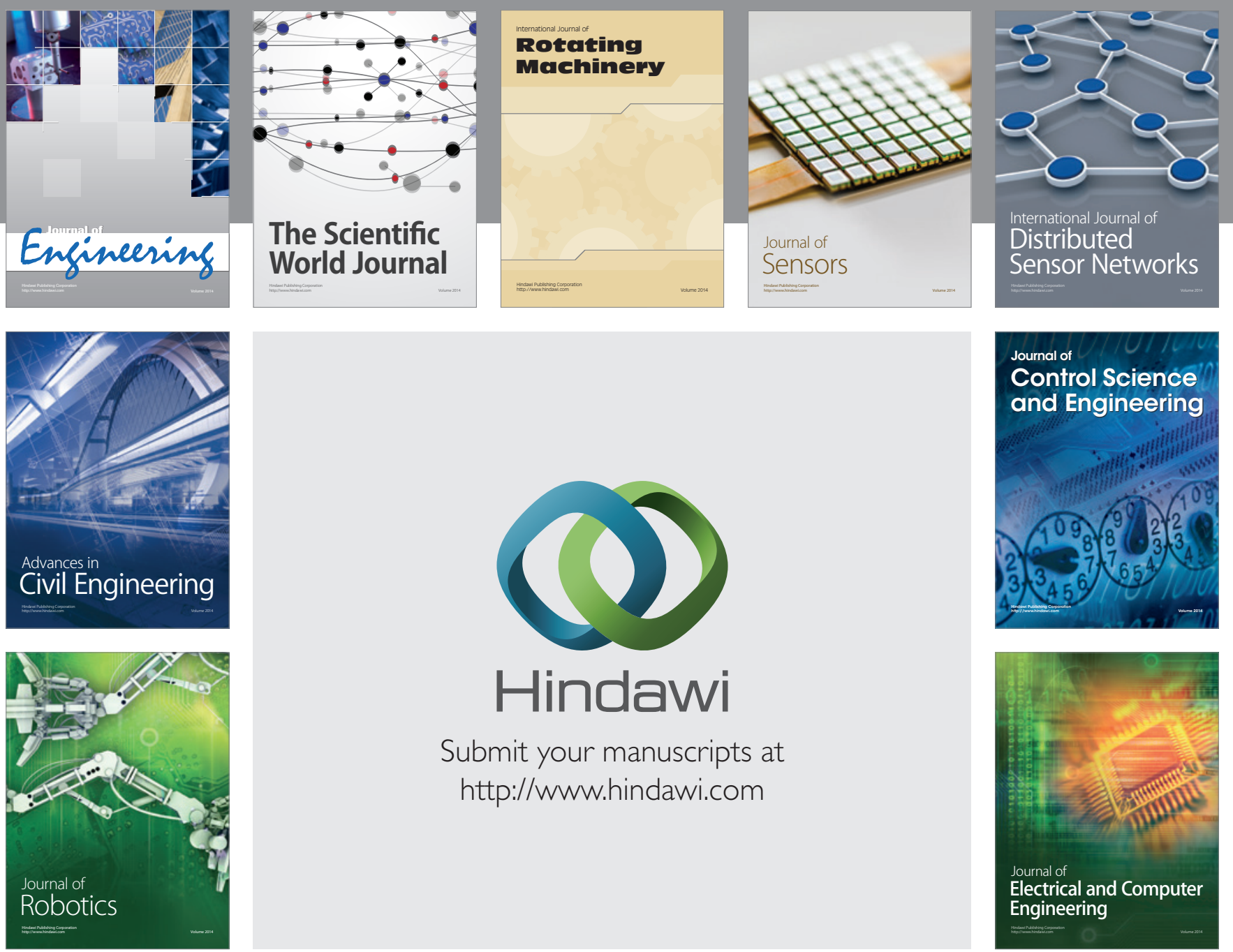

Submit your manuscripts at

http://www.hindawi.com
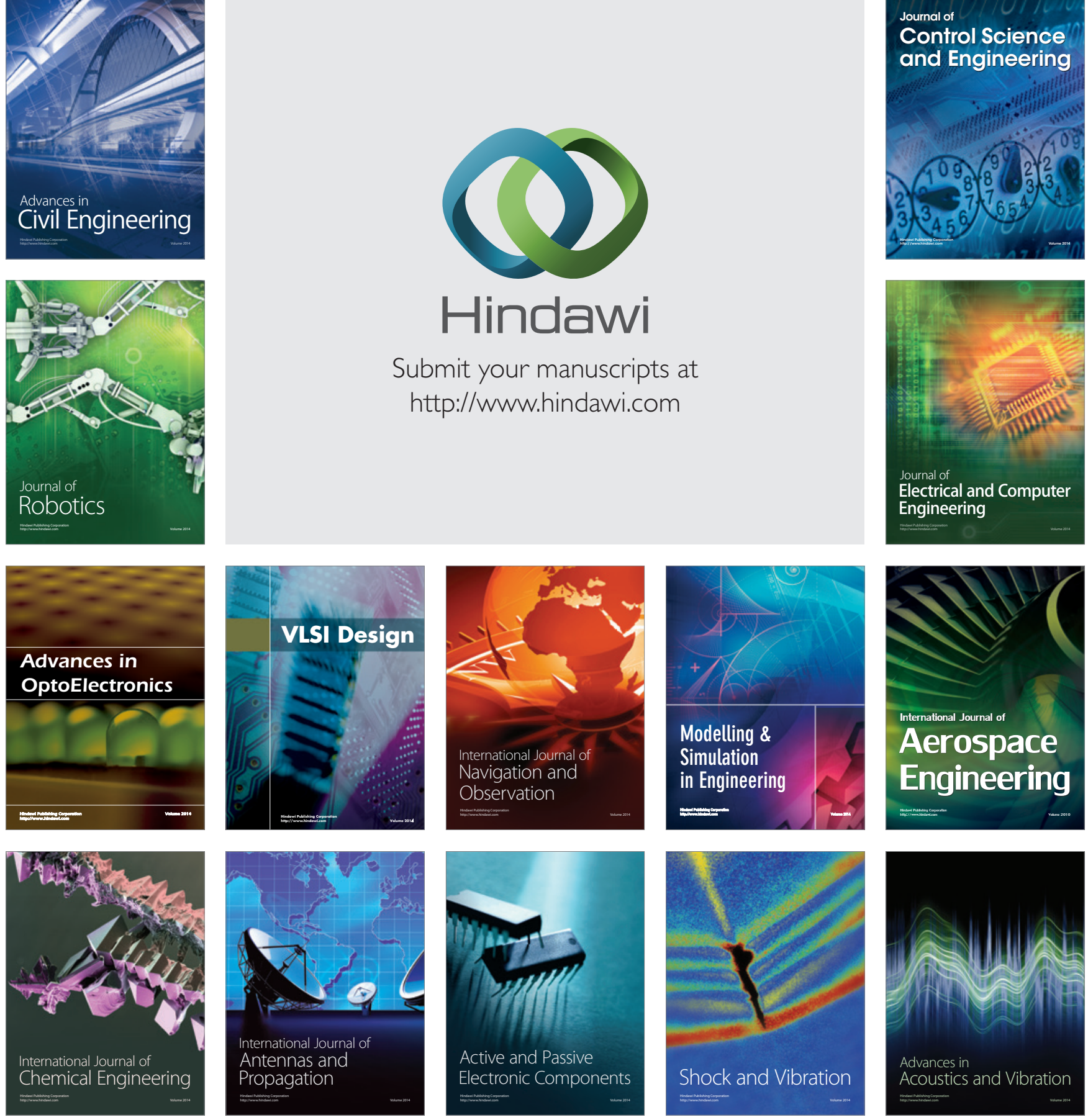